\title{
Numerical Investigation of Shock-Train Response to Inflow Boundary-Layer Variations
}

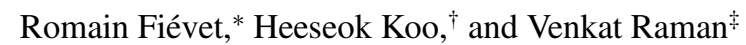 \\ University of Michigan, Ann Arbor, Michigan 48109-2102 \\ and \\ Aaron H. Auslender \\ NASA Langley Research Center, Hampton, Virginia 23681
}

DOI: $\underline{10.2514 / 1 . J 055333}$

\begin{abstract}
A dataset of normal shock trains in a rectangular cross-section channel has been created from direct numerical simulations in an effort to quantify the impact of inflow confinement ratio on the shock-train structure. To this end, the inlet boundary-layer momentum thickness was varied while the bulk inflow and outflow conditions remained constant. The simulations show that the shock train is displaced upstream as the inflow confinement ratio increases. Also, an increase in boundary-layer momentum thickness results in a reduction of the normal-like portion of the lambda-shock structures in the channel core. This leads to more numerous but weaker bifurcating shocks as well as an increase of the shock-train length. When the inflow boundary-layer thickness is varied temporally, the complex shocktrain response depends on the excitation frequency. A resonant frequency is observed when different components of the shock train exhibit the highest amplification in terms of pressure jumps. Further, the domain upstream of the leading shock acts as a low-pass filter, which has the end result of limiting the axial shock-train motion. Nevertheless, even a small oscillation in boundary-layer momentum thickness of $0.45 \mathrm{~mm}(0.6 \%$ of the channel height) is seen to increase the shock-train length by two orders of magnitude $(4 \mathrm{~cm})$.
\end{abstract}

\section{Introduction}

$\mathbf{I}_{\mathrm{s}}^{\mathrm{N}}$ $\mathrm{N}$ DUAL-MODE scramjet engines, the precombustion isolator section contains a shock train characterized by a core supersonic flow and a subsonic boundary-layer-dominated flow. Shock trains provide compression through not only a series of shock structures but also through flow confinement due to the growth of the near-wall subsonic region. The pressure and temperature gain through this shock train is critical for ensuring efficient combustion farther downstream in the flow path. Hence, understanding the stability of shocks in confined supersonic flows is of interest for dual-mode operation.

In constant-area channels, a supersonic flow with a turbulent boundary layer exhibits a range of flow structures based on the inlet Mach number $M a$ [1] . At low supersonic speeds $(M a<1.2)$, a normal shock transitions the flow to a subsonic state. This normal shock becomes increasingly curved with increase in flow speed, and at velocities greater than $M a=1.5$, the single normal shock bifurcates and forms a series of shocks, referred to as a shock train. More importantly, the turbulent boundary layer undergoes separation leading to an axial reduction in the size of the core supersonic flow. This flow restriction, or confinement, through the formation of a subsonic region is critical in determining the structure of the shock train. Its effect on shock trains is directly related to the incoming boundary-layer properties. For instance, Carroll and Dutton [2] have shown that as the ratio of inflow boundary-layer thickness to duct height increases, both the number of shocks and the spacing between the shocks increases. At the same time, the bifurcation associated with the leading shock reaches more toward the center of the flow

Received 4 May 2016; revision received 17 February 2017; accepted for publication 21 February 2017; published online 12 May 2017. Copyright (C) 2017 by Romain Fiévet. Published by the American Institute of Aeronautics and Astronautics, Inc., with permission. All requests for copying and permission to reprint should be submitted to CCC at www.copyright.com; employ the ISSN 0001-1452 (print) or 1533-385X (online) to initiate your request. See also AIAA Rights and Permissions www.aiaa.org/randp.

*Ph.D. Student, Department of Aerospace Engineering. Student Member AIAA.

${ }^{\dagger}$ Postdoctoral Research Fellow, Department of Aerospace Engineering. Member AIAA.

${ }^{\ddagger}$ Associate Professor, Department of Aerospace Engineering. Associate Fellow AIAA.

${ }^{\S}$ Research Aerospace Engineer, Hypersonic Propulsion Branch. with an increase in confinement ratio, defined as the ratio between the inlet boundary layer and channel half-height. Further, the effect of confinement ratio decreases as the Mach number increases.

Such supersonic duct flows have been studied using both experimental [1-21] and computational [22-37] approaches. The experimental studies have mainly used pressure measurements, laser Doppler velocimetry, and schlieren images to reconstruct the shocktrain structure. Waltrup and Billig [4] analyzed pressure traces to propose an empirical relation between shock-train length and wall pressure. In that work, the shock-train length was found to have a $R e_{\theta}^{1 / 4}$ dependence, where $R e_{\theta}$ is the momentum-thickness-based Reynolds number at the inlet of a cylindrical isolator section. This correlation was later adapted to rectangular isolators with a $\operatorname{Re}_{\theta}^{1 / 5}$ dependence [3]. Carroll and Dutton [2, $\underline{3}$ ] have observed experimentally the effect of confinement ratio on the shock-train shape using schlieren imaging. More recent measurements [7] show that the shock structures are inherently unsteady, with the oscillation amplitude and frequency increasing with inflow Mach number.

Other studies $[12,17-19,36,37]$ have focused on the physics of the unstart process, where the shock train is completely dislodged by an unsupportable pressure rise across the isolator section. Such instability can be caused either by upstream (change of flight conditions) or downstream (unstable combustion) perturbations. Typically, downstream perturbations have been simulated by changing the isolator exit pressure over time at a particular excitation frequency [12,17-19]. Klomparens et al. [17] have identified a hysteresis effect during the cyclic motion that causes the shock train to travel along different paths during the upstream and downstream parts of the cycle. Bruce and Babinsky [18] found that the shock oscillations induce a corresponding change of relative Mach number, which changes both its shape and the wall pressure profile, depending on the direction of motion of the shock.

Understanding the sensitivity of shock trains to the near-wall flow is also useful for controlling the unstart process. In particular, perturbations to the boundary layers through actuation mechanisms can be used to delay unstart. For instance, Hutchins et al. [19] studied a Mach 1.8 channel flow, where both the inlet total pressure and the backpressure can be altered separately. This configuration was used to build a nonlinear transfer function capable of predicting the timedependent shock-train location when subjected to both upstream and downstream pressure instabilities. Valdivia et al. [12] used the same experimental configuration to demonstrate active control of the 
shock-train position in a situation of imminent unstart. Using sidewall passive vortex injectors, an overpressure of $32 \%$ compared to the injector-free case was achieved without unstart. Similar boundarylayer modifications have been used to modify the shock train in other studies [9,14]. For instance, Do et al. [9] studied unstart in a Mach 5 wind tunnel by impinging the flow with a wall-normal injector. It was found that symmetric, thin, and laminar boundary layers were able to sustain this flow blockage longer than thick and turbulent boundary layers.

In addition to such experiments, a number of computational studies have also been used to study isolators. Such simulations are essentially of two kinds. The first kind uses an inlet ramp to create an initial incident shock that is relatively fixed in time [22-24,35,38]. This yields a steady attached shock train, which is fixed to the foot of the first reflected shock and can be expected to be adequately described by inviscid theory. Of course, farther downstream, the reflected shocks are weaker and are affected by the turbulent flow structure inside the isolator. Koo and Raman [22] used a large-eddy simulation (LES) to simulate a Mach 5 supersonic inlet isolator with unstart, which was caused by an increase in backpressure at the outlet. Overall, they concluded that the LES approach, with relatively simple wall models, is able to capture the overall shock structure for both static and unstart cases. However, there was a significant difference in the time scales associated with unstart, where the LES predicted a faster shock propagation speed compared to the experiments. Perhaps most relevant to the current study, Su et al. [24] simulated a ramp-based inlet of a vehicle flying at high altitude and at Mach 6. In that work, a varying backpressure condition was applied at the outlet. It was observed that the shock-train oscillation amplitude was the highest for the smallest frequency simulated and that increasing the dynamic pressure oscillation amplitude increased the corresponding wall pressure oscillation amplitude.

The second kind of isolator simulations involves a normal shock train [25-33], which is more relevant to the dual-mode scramjet regime. Here, the initial shock bifurcation is caused by flow confinement, which requires either a numerical backpressure condition at the outlet or the inclusion of the combustor in the computation domain. Reynolds-averaged Navier-Stokes simulations (RANS) [25], large-eddy simulations (LES) [27-29,31,34], and hybrid models $[32,33]$ have been used. In general, LES and hybrid RANS/LES approaches have been fairly successful in capturing experimental measurements, as opposed to RANS computations [39]. In particular, wall-modeled approaches or techniques that contain no special treatment for the shock-boundary-layer interaction still predict the shock-train structure reasonably well, although wall modeling was found to slightly improve predictive accuracy [31]. Such studies also demonstrated that boundary-layer modification can alter shock-train location. For instance, wall cooling reduced the distance between the shocks [31].

Overall, these experimental and computational studies highlight the key role of the boundary layer in determining the shock-train structure and stability. The focus of this work is in understanding the response of the shock train to dynamic modification of the incoming turbulent boundary layer in a rectangular isolator. To limit the impact of any modeling errors, a high-resolution numerical approach is followed, where the smallest near-wall features are fully resolved. Using this approach, the changes in the shock-train structure to modifications in the boundary layer are studied. The dynamic response is then studied on a coarser mesh to resolve long time-scale motion of the shock-train structure. The rest of the sections are organized as follows. Section II discusses the flow configuration and numerical details. Section III presents the results on the effect of boundary-layer thickness on shock-train evolution as well as a comparison with experimental data. Section IV is dedicated to temporal effects on shock-train motion, as the inflow boundary-layer momentum thickness oscillates at different frequencies. In particular, the role of the shock train in processing upstream disturbances is analyzed in detail.

\section{Flow Configuration and Numerical Details}

The simulation configuration is chosen to replicate the University of Michigan expansion tunnel facility, where an experimental study on the effect of backpressure variations on shock-train behavior is being conducted $[16,17]$. The simulation domain consists of a $69.8 \times$ $57.2 \mathrm{~mm}$ constant-area rectangular channel to which a backpressure is applied at the outlet as a numerical boundary condition. Figure 1 presents a qualitative view and some perspective of the computational domain and typical flow structures. The inflow is at Mach 2 and is injected as a turbulent channel flow at pressure $P_{a}$. The backpressure $P_{b}$ is fixed to $46,200 \mathrm{~Pa}$ corresponding to about $70 \%$ of a normal shock pressure rise for this Mach number. The channel half-height $h=34.9 \mathrm{~mm}$ is used as a reference length throughout the paper. The computational domain spans $856 \mathrm{~mm}$ in the streamwise direction (roughly $25 h$ ), which is sufficient to fully accommodate the shock train for the range of conditions studied here. The domain is discretized using an orthogonal grid system of various resolutions as detailed in Table 1 . The grid cells are clustered near the walls such that the spatial resolution based on wall units is $\{\Delta x+=12.6$, $\Delta y+=0.69-14.7, \Delta z+=0.69-14.7\}$. The wall viscous length scale was computed to be $22 \mu \mathrm{m}$ based on the inflow boundary-layer statistics shown in Fig. 2 .

As previously discussed, the focus of this study is to understand the impact of inflow boundary-layer conditions on shock-train formation and response. For this purpose, three different inflow boundary layers
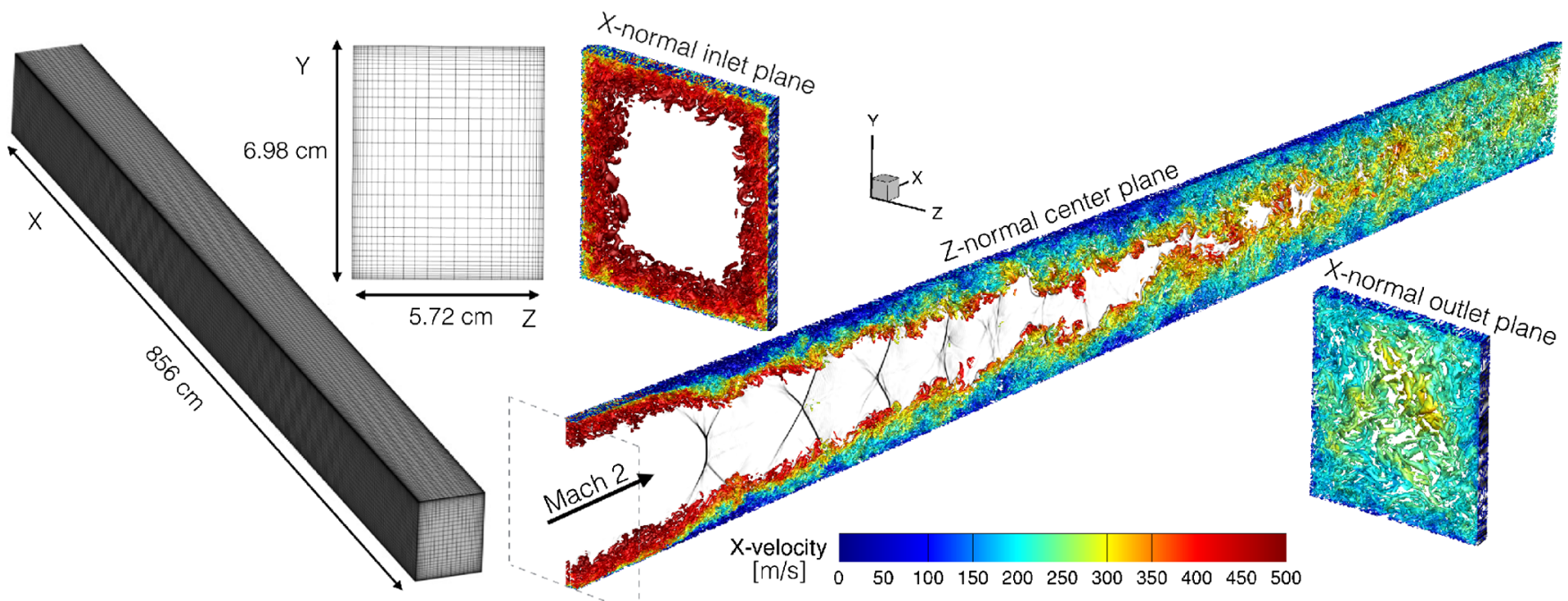

Fig. 1 Computational domain and grid with 1/8th cells in each direction (left). Isocontours of second invariant of the velocity gradient tensor colored by streamwise velocity with density gradient in black (right). 
Table 1 Different grid resolutions

\begin{tabular}{lccccccc}
\hline \hline Grid & $N_{x}$ & $N_{y}$ & $N_{z}$ & $\Delta x+$ & $\begin{array}{c}\text { Minimum } \\
\Delta y+/ \Delta z+\end{array}$ & $\begin{array}{c}\text { Maximum } \\
\Delta y+/ \Delta z+\end{array}$ & $\begin{array}{c}\text { Number } \\
\text { of cells } \\
<10 y+\end{array}$ \\
\hline R1 & 1024 & 160 & 128 & 35.4 & 1.90 & 41.2 & 4 \\
R2 & 2048 & 320 & 256 & 17.7 & 0.95 & 20.6 & 8 \\
R3 & 3072 & 480 & 384 & 12.6 & 0.69 & 14.7 & 13 \\
\hline \hline
\end{tabular}

generated from an auxiliary channel flow simulation are used. The inflows are generated from an auxiliary simulation on the same domain as the main channel simulation, but without a numerical backpressure. The domain has streamwise periodic boundary conditions, which simplifies boundary-layer development, and a grid resolution of $\{\Delta x+=17.7, \Delta y+=0.95-20.6, \Delta z+=0.95-20.6\}$. Starting from a uniform flow, the governing equations are evolved using a fourth-order Runge-Kutta temporal scheme, sixth-order compact scheme for spatial discretization, and an eighth-order implicit filter with hyperviscosity. The turbulent boundary layer grows to the target height, at which point the flowfield is sampled with planes of data written to a data file. Because the turbulent boundary layer grows during this time, the data are collected only for a short time of $1 / 4$ flowthrough times. This approach allows inflow data that are continuous in space and time (within numerical accuracy) to be generated. Further, no numerical shocks are observed in the main simulation, which can arise from discontinuous inflow conditions that are patched in time. The inflow conditions and the corresponding parameters are shown in Table 2 . The resolution was carefully chosen such that no extraneous compression shocks are generated at the inlet due to a mismatch between the isolator and channel computational grids. Figure 2 presents the wall-normal time-averaged velocity and turbulence intensity profiles for all inflows. It is seen that the Van Driest transformed velocity profiles are similar to conventional incompressible turbulent boundary-layer flows, which is a known result [40] and compares well with similar flows in the literature, such as the Mach 2.32 and $R e_{\theta}=4450$ direct numerical simulation (DNS) of Martin [40]. Likewise, the wall-normal density-weighted Reynolds stresses profiles at the bottom wall compare favorably well with other profiles in the open literature considering the differences in viscosity and Mach and Reynolds numbers $[28,34,40,41]$. Closer to the corners, the interaction of the boundary layers will cause the profiles to differ from the conventional log-law profiles.

The simulations were performed using the in-house compressible flow solver UTComp, which has been extensively verified and validated $[23,42-45]$. The solver uses a finite difference fifth-order weighted essentially non-oscillatory Lax-Friedrichs (WENO LLF) scheme with characteristics reconstruction to compute the fluxes $[46,47]$. A fourth-order central scheme is used for the viscous and diffusion terms, and the walls are treated adiabatically. The viscosity is determined using Sutherland's law and is multiplied by 4 to obtain a more tractable Reynolds number. It is estimated that using standard
Table 2 Flow conditions for the three different inflow considered

\begin{tabular}{lcccccccc}
\hline \hline Case & $P_{a}, \mathrm{kPa}$ & $T, \mathrm{~K}$ & $M a$ & $\delta, \mathrm{mm}$ & $\delta / h$ & $\delta^{*} / h$ & $\theta, \mathrm{mm}$ & ${R e_{\theta}}$ \\
\hline $\mathrm{A}$ & 14.1 & 170.0 & 1.97 & 8.8 & 0.25 & 0.046 & 1.3 & 4530 \\
$\mathrm{~B}$ & 14.5 & 171.0 & 1.97 & 9.8 & 0.28 & 0.070 & 1.8 & 6380 \\
$\mathrm{C}$ & 14.8 & 172.0 & 1.96 & 11.9 & 0.34 & 0.083 & 2.2 & 7900 \\
\hline \hline
\end{tabular}

air viscosity would bring the wall unit down to about $9 \mu \mathrm{m}$, increasing the computational costs by a factor of 36 and requiring an 8.2-billion-cell grid to achieve the same resolution. The thermal diffusivity is obtained using a constant Prandtl number of 0.72 . Further details on the flow solver are provided in $[22,46]$. The code uses domain-decomposition-based parallelization. Each case was initialized on grid R1 with respective inflow conditions in the first half of the channel and post-pressure-rise conditions in the second half. Each case was computed until a stable shock train was constructed; its position along the channel was observed to be fully converged over $30 \tau_{c}$. Each case was then interpolated on grid R2, which was further run on 4000 cores for $120 \mathrm{~h}$ over $8 \tau_{c}$. During the R2 run, the shock train became unsteady for all cases. It first relaxed downstream, slowed down, then moved steadily upstream at a constant velocity of $1.5 \%$ mean centerline velocity. The shock trains were then interpolated on grid R3 and run for an additional $24 \mathrm{~h}$ on 8000 cores over $1.5 \tau_{c}$. Statistics were then sampled over $0.5 \mathrm{~ms}$ on $\mathrm{R} 3$, which corresponds to a quarter of a flow-through time scale $\tau_{c}\left(\tau_{c}=2.05 \mathrm{~ms}\right)$ based on the integrated centerline velocity, or one inflow period. Although every simulation ran over multiple $\tau_{c}$, it was found that sampling statistics over a longer time would decrease the shock resolution due to the unsteady position of the shock train. The simulations ran with a Courant-Friedrichs-Lewy number of 0.9, giving a time step of about $32 \mathrm{~ns}$ for the finest grid.

A grid convergence analysis was performed with conditions corresponding to case B and is shown in Fig. 3 . Time-averaged profiles for the stable R1-grid sampled over the whole run $\left(30 \tau_{c}\right.$, i.e., greater than $0.06 \mathrm{~s}$ ) are also shown. The relative L2-norm error of R1 and $\mathrm{R} 2$ wall pressure profiles with respect to $\mathrm{R} 3$ are 2.01 and $0.32 \%$. The convergence from R2 to R3 of both wall and centerline pressure profiles are excellent. Interestingly, even the coarsest grid (R1) reproduces the bulk of the physics by resolving most shock cell sizes and location as well as capturing the time-averaged wall pressure growth rate. However, R1 is unable to correctly resolve the leading cell's compression and expansion wave strengths. The underresolution of the successive compression/expansion cycles leads to a roughly 3-4 h shorter shock train based on the pressure timeaveraged profiles. The $\mathrm{R} 3$ simulation achieves both convergence and meets the resolution requirement for a wall-confined DNS [48]: $\Delta x+<15, \min (\Delta y+)<1$, and at least three cells included within a distance of $10 y+$ from all walls (in our case, the spanwise direction $z+$ is also a wall-normal direction with similar resolution). In fact, the maximum $\Delta y+/ \Delta z+$ throughout the inlet boundary layer are 6.9,
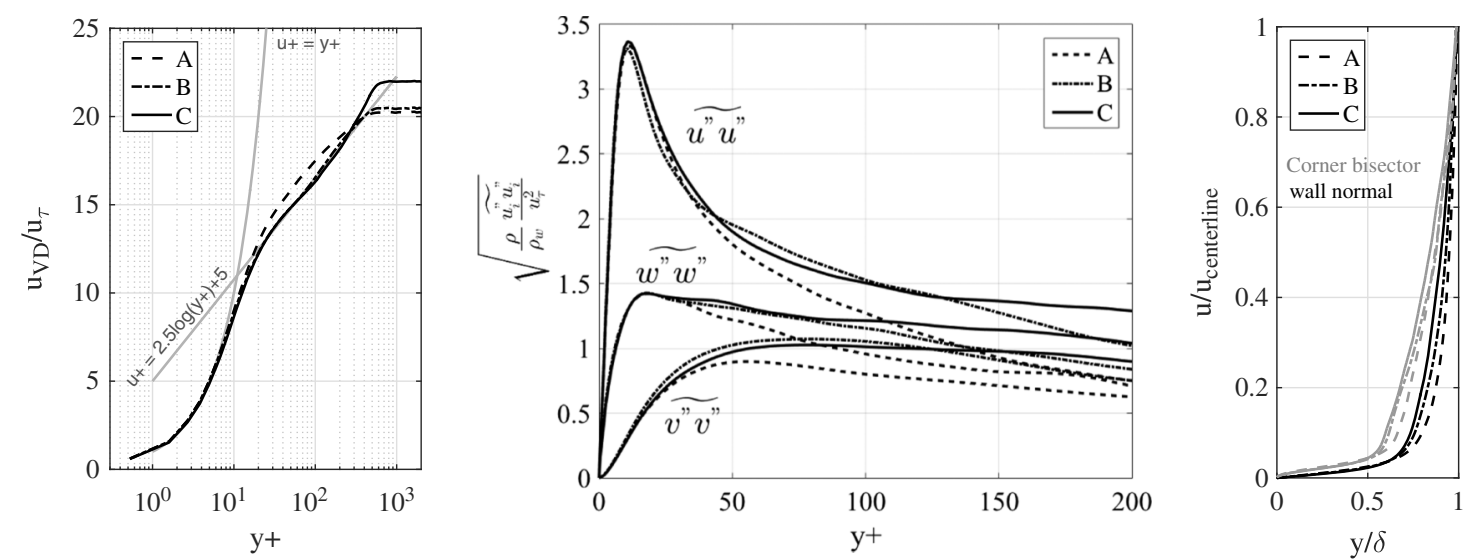

Fig. 2 Turbulent boundary-layer profiles for all cases: wall-normal Van Driest transformed velocity profiles (left), wall-normal density-scaled turbulence intensity profiles (middle), and corner bisector and wall-normal Reynolds-averaged velocity profiles (right). 

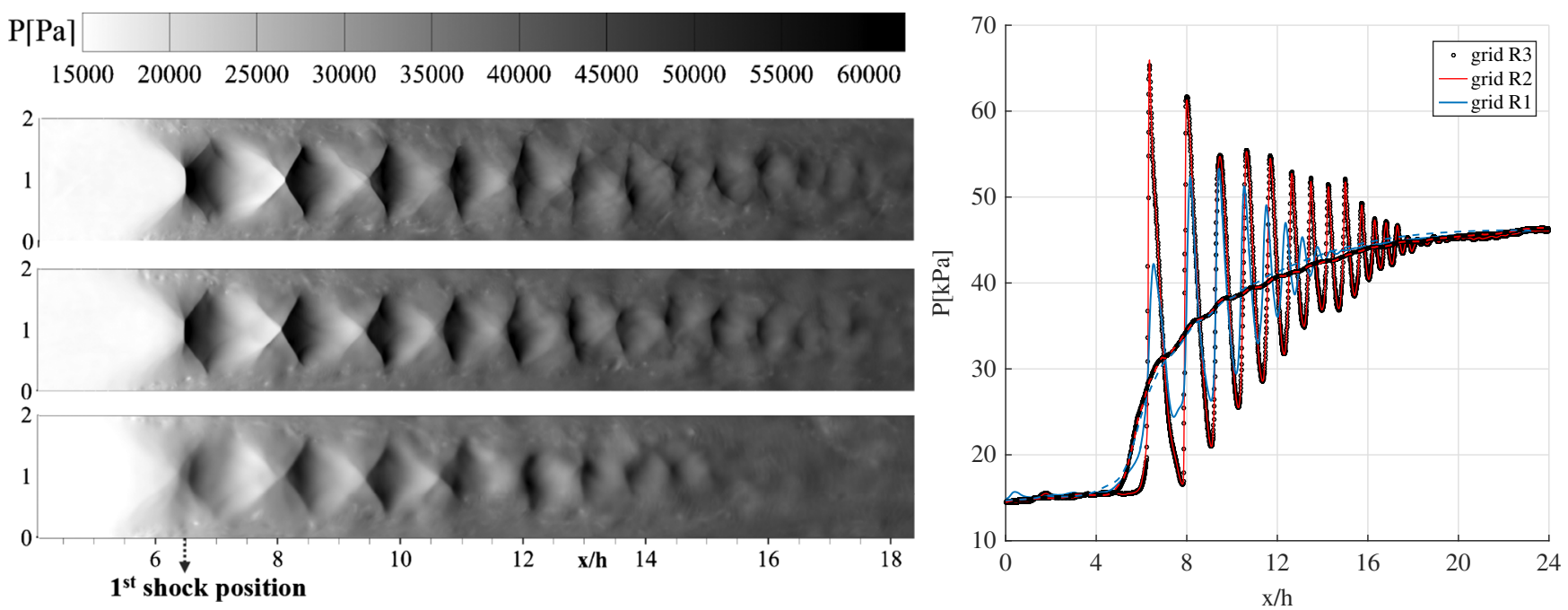

Fig. 3 Typical static pressure snapshots for grid R3, R2, and R1, top to bottom (left). Centerline (solid) and wall (dashed) time-averaged static pressure profiles for all grids (right).

7.6, and 8.8y+ for cases A, B, and C, respectively. The R3 simulation will be referred to as a DNS throughout the paper.

Additionally, to ensure that the simulations capture the general trends observed in the experiments, direct comparison with the experimental data of Klomparens et al. $[16,17]$ is presented here. Figure 4 shows the time-averaged DNS pressure profiles compared with experimental measurements. In these plots, $P_{0}=P\left(x_{0}\right)$ denotes the pressure on the lower wall preceding the location of pressure rise $x_{0}$. In the experiment, the shock-train inflow confinement ratio is increased/decreased by displacing the train downstream/upstream in the tunnel. This motion is achieved by changing the backpressure ratio through a control valve downstream. From the available data, case $\mathrm{B}$ is the closest to the experimental conditions : $\delta / h=0.27$ for both and $P_{b} / P_{a}=3.17$ and 3.18 for the experiment and the DNS, respectively. It should be noted that, because of the artificial increase in viscosity in the simulations, the inflow momentum thickness is twice as large in the experiments for the same confinement ratio. The bump observed in the experimental profile around $\left(x-x_{0}\right) / h=-1$ is an uncanceled wave from the nozzle expansion process. As seen in Fig. 4, the simulations compare well with experiments. Even so, there is a steeper pressure rise in the simulation compared to experiments. This is consistent with other simulation data (Morgan et al. [28] and Roussel et al. [34]) also. One possible reason is the time duration of averaging. In the experiments, the data were averaged over $10-15 \mathrm{~s}$, over which low-frequency oscillations of the shock structure were observed. The simulations were averaged over a much shorter time. The low-frequency oscillations were observed to have a broad spectral response, with a frequency between 90 and $120 \mathrm{~Hz}$. It is likely that these oscillations act as a moving bandwidth averaging

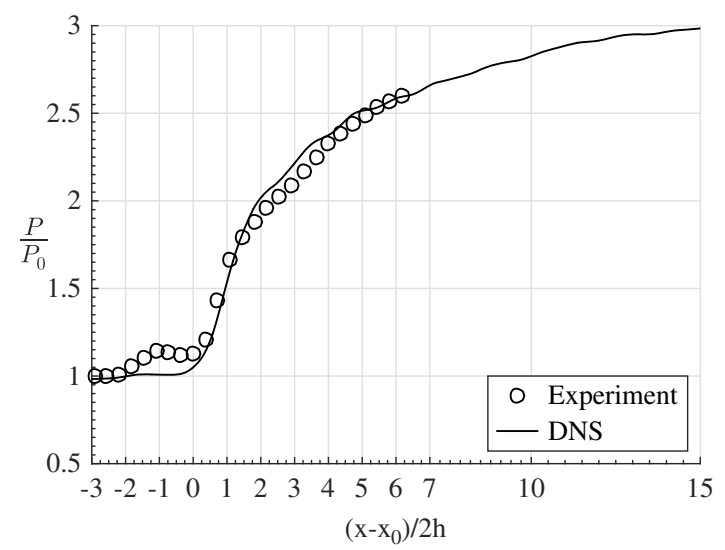

Fig. 4 Time-averaged pressure profiles along the wall from simulation and experiment for case $B$. filter, leading to a more gradual pressure rise in experiments compared to the simulations.

\section{Effect of Inflow Boundary-Layer Thickness}

\section{A. Instantaneous Snapshots}

The three cases (A, B, and C, see Table 2) were simulated using the procedure described in Sec. II. Figures 5 and 6 show the DNS instantaneous Mach number and density gradient magnitude contours for all cases on the center plane in the spanwise direction. Similar to experimental predictions, a series of shock structures is present. Once the primary shock is formed, the boundary layer thickens, leading to an increasing volume of subsonic flow with axial distance. Note that the density gradients across the shock structure are roughly equal for all cases, indicating that the growth of the subsonic region plays a bigger role in affecting the axial compression rate. Past the terminating (last) shock, a mixing region is observed, where both subsonic and supersonic flow are present. Overall, the simulation reproduces the essential features of a shock train. The observed trend is that a higher confinement ratio moves the shock train upstream.

\section{B. Shock-Train Structure Dependence on Convective Velocity}

The equilibrium location of the shock train changed when interpolating from the coarse grid R1 to the finer grids R2 and R3, and all shock trains started moving. The equilibrium position of the shock train depends on the balance between the backpressure force (which pushes the shock train upstream) and the wall shear stress (which attaches the shock train to the wall). Because the boundary layer is better resolved on the finest grids, that location is subject to change during grid refinement. When interpolating from grid R1 to R2, we observed a slow steady displacement upstream of the shock train for case B. For cases A and C, the shock train first relaxed downstream, slowed down, then moved steadily upstream. These low-speed and large-scale displacements from the previous R1 steady location existed for many flow-through times over the whole R2-simulation as seen in Fig. 7. The shock train is still steadily moving upstream by the end of the $\mathrm{R} \overline{2}$ and $\mathrm{R} 3$ simulations, with a converged convective speed comprised between 7 to $9 \mathrm{~m} / \mathrm{s}$, which corresponds to about $1.5 \%$ of the bulk inflow velocity. Consequently, even with the long simulation times considered here, the statistics cannot be assumed to be fully converged because very low-speed dynamics may not have been completely considered. Hence, as mentioned in Sec. II, the DNS statistics are sampled over $0.5 \mathrm{~ms}$, which corresponds to a quarter of a flow-through time scale $\tau_{c}\left(\tau_{c}=2.1 \mathrm{~ms}\right)$. Such unsteadiness is likely be a consequence of the grid refinement and has been previously observed by Morgan et al. [28], who had to decrease the backpressure by $15 \%$ to keep the shock train idle on their finest grids. Alternatively, this could have a physical relevance because oscillatory behaviors are 


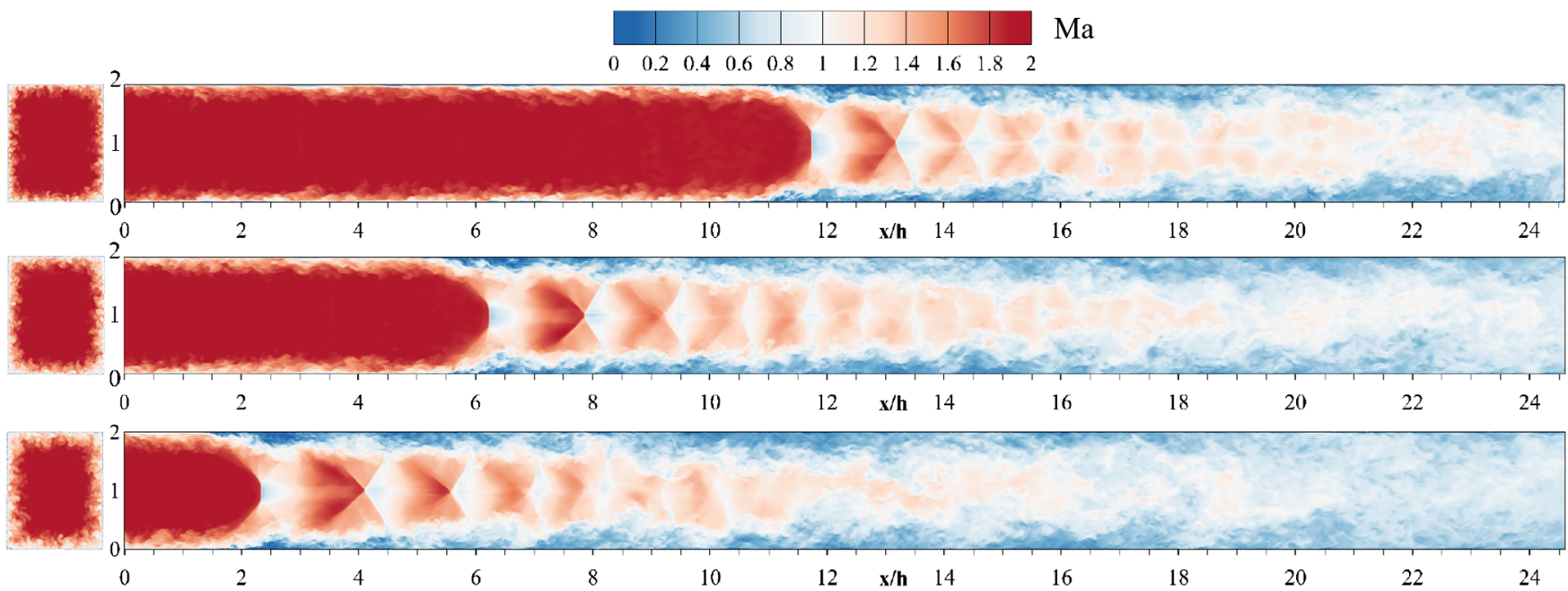

Fig. 5 Instantaneous snapshots of Mach number for case A (top), case B (middle), and case C (bottom), with the inlet plane on the left side.

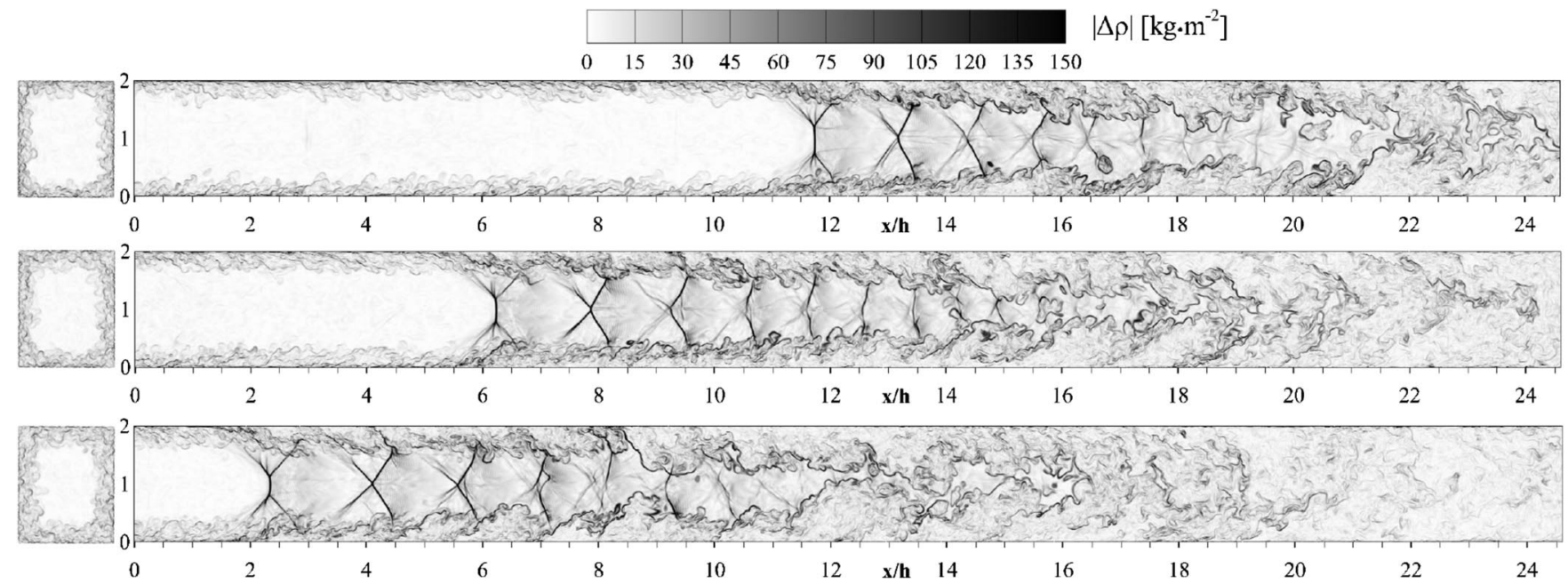

Fig. 6 Instantaneous snapshots of density gradient magnitude for case A (top), case B (middle), and case C (bottom), with the inlet plane on the left side.

common in shock-boundary-layer interactions [41,49-51]. Regardless of the cause, the direction of shock-train motion interestingly affects its structure appreciably. This change in structure is shown in Fig. 7, where 16 frames are equally spaced in time and span a total time of $2 \tau_{c}$ for case A. The contours of numerical schlieren images highlight both the shape of the shocks and the turbulent boundary layer, giving insight into their coupling mechanics. Indeed, the magnitude of the boundary-layer separation depends on the pressure gradient between the wall and the centerline and is therefore a good indicator of the first shock strength. As can be seen from Fig. 7, the shock train first moves downstream toward the outlet and comes back upstream against the flow. When the shock train moves with the flow, its relative speed decreases, which weakens the shocks, whereas with an upstream shock motion, the relative Mach number increases, which strengthens the shocks. This causes the appearance of slip lines along the normal-like portion of the lambda shocks at the bifurcation points with the expansion waves [2]. A crude evaluation of the apparent Mach number evolution can be made, assuming from Fig. 7a sinusoidal oscillation of amplitude of 1 channel height and of period $T=2 \tau_{c}$. This leads to a relative convective speed of maximum amplitude $\pm 2 \pi h / T$, resulting in a range of apparent Mach number $M a_{\text {app }} \in[1.83 ; 2.17]$, oscillating around the bulk Mach number $M a=2$. This broad range of apparent Mach number changes the nature of the shock train from a normal shock train to an oblique shock train type (smaller normal part in the leading shock, thicker boundary layer, and larger distance between shocks) [1]. In Fig. 7, frames 1-4 correspond to a weak structure, 5-8 to a steady strand, and 9-16 to a strong shock structure. A comparison between frames 1 and 16 validates the previous statements because both the distance between shocks and the turbulent boundary-layer thickness increase, which is characteristic of a higher Mach number shock train. Because of the large time scales involved with this phenomenon, statistics might differ based on whether the samples were taken in the oblique/weak or normal/strong shock-train instances. Similar changes in shock structure have been observed experimentally [18], albeit with smaller oscillation amplitude in a lower Mach number flow. These observations are parts of the motivation to investigate the shock-train response to forced oscillations in Sec. IV.

\section{Time-Averaged Contours}

Figures $\underline{8}$ and $\underline{9}$ show the time-averaged statistics of the Mach number and pressure fields, respectively, for the three different inflow confinement ratios. These statistics correspond to the last $0.5 \mathrm{~ms}$ $\left(\tau_{c} / 4\right)$ of the DNS run. Case A's leading lambda shock presents a larger normal-like portion than case $\mathrm{C}$, resulting in a larger subsonic region behind it. This suggests that the first shock strength depends directly on the confinement ratio. It is found that a thinner boundary layer leads to larger pressure gradient near the wall, which accelerates boundary-layer detachment.

This feature appears clearly when plotting wall and centerline pressure signals on the center plane in the spanwise direction. Figure 10 shows the centerline and wall pressure traces, whereas the middle figure in Fig. 11 shows the wall pressure growth along the pseudoshock region. The shock-train length is then defined based on the location where $90 \%$ of the compression is reached and is denoted by $x_{s t}$. Overall, the same compression ratio is achieved over a shorter 


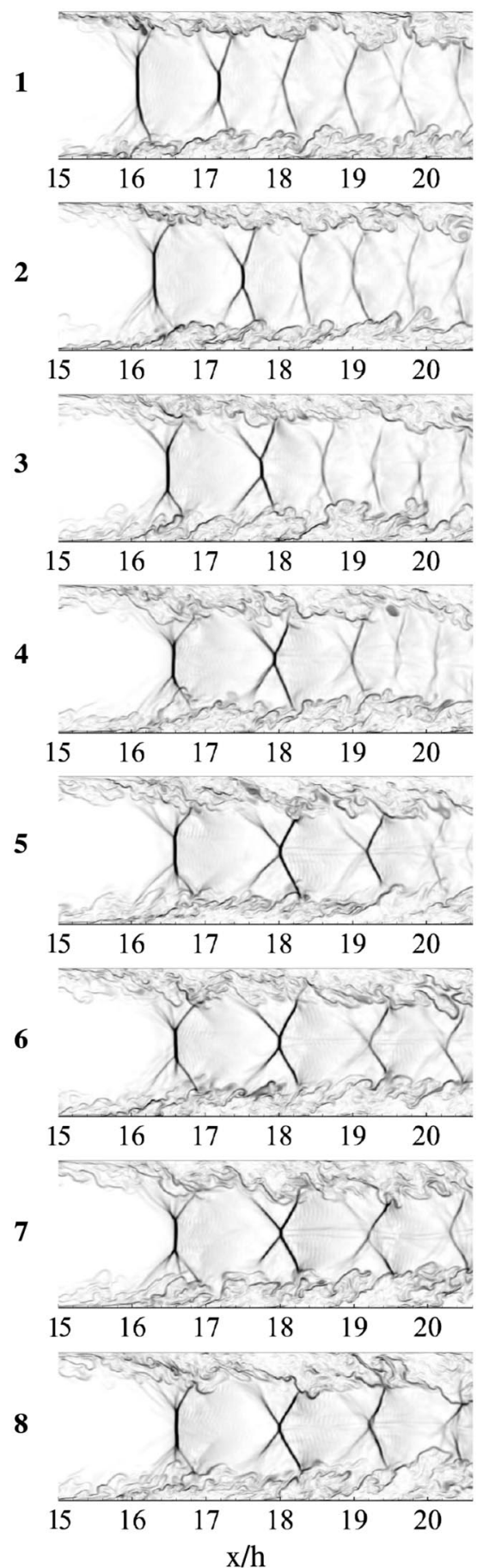

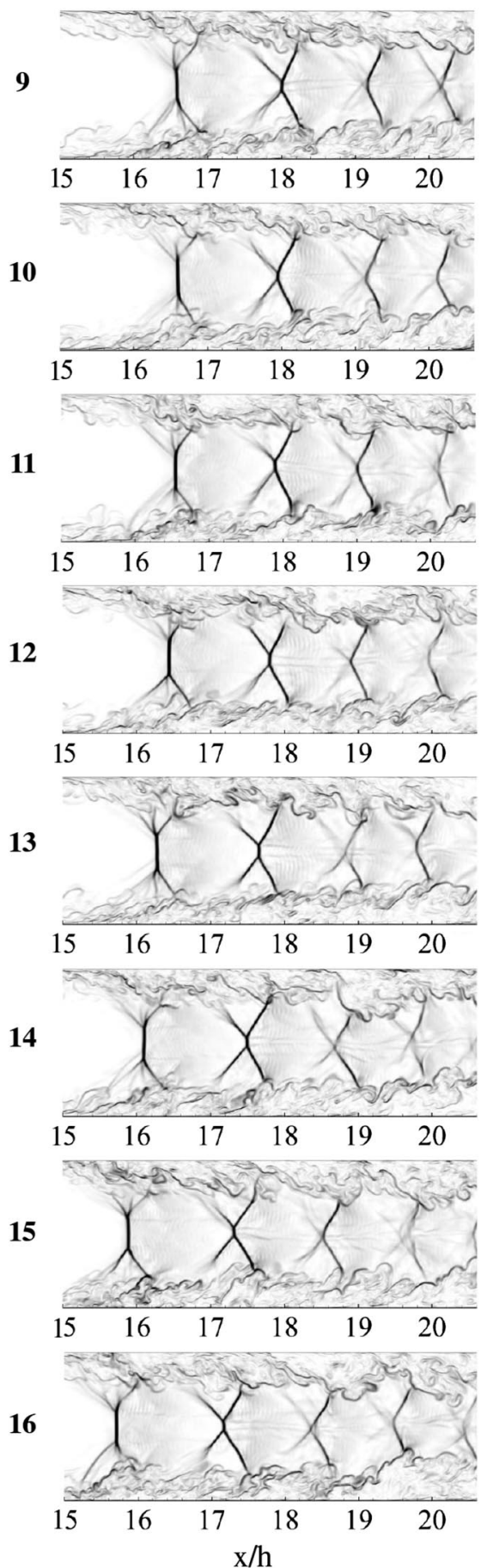

Fig. 7 Snapshots of density gradient of the shock-train foot for case A on grid R2 at 16 different times equally spaced over a duration of $2 \tau_{c}$.

length for a shorter inlet confinement ratio, with the wall pressure showing a steeper rise for smaller inlet $\delta / h$. To estimate the axial rate of compression, a quantity $\Sigma$ is defined such that

$$
\Sigma(x)=\int_{(y z)} \frac{P(x)-P_{a}}{P_{b}-P_{a}} \mathrm{~d} y \mathrm{~d} z
$$

It is seen in the middle figure in Fig. 11 that the first cell compression is stronger and shorter. This difference is further increased for the following cells, therefore shortening the shock train. This metric shows a linear correlation with inflow momentumthickness-based Reynolds number as seen on the right figure.

Based on both instantaneous and time-averaged results, the following dependence of the shock-train structure on the inlet confinement ratio can be elucidated. From case A to case $C$, the confinement ratio (or boundary-layer thickness) increases. The leading shock is bifurcated with a normal-like portion and a lambda shock that interacts with the boundary layer. Because the Mach number is the same for all simulations, the angle of the oblique foot of the lambda shock remains the same as well. As the confinement ratio increases, the bifurcation point moves toward the center of the flow, leading to a smaller normal-like portion. Because normal shocks provide higher compression, this reduction in size leads to a weaker compression across individual shock structures in the shock train. At the same time, as the bifurcation point moves toward the center of the channel, the back foot (downstream part) attaches to the boundary layer farther downstream, delaying the appearance of the expansion wave and the subsequent shock structures. Consequently, increasing the confinement ratio increases the diamond-patterned cell size but decreases compression and flow deceleration. Past the first shock, reattachment causes the boundary layer to regenerate, and the influence of the 

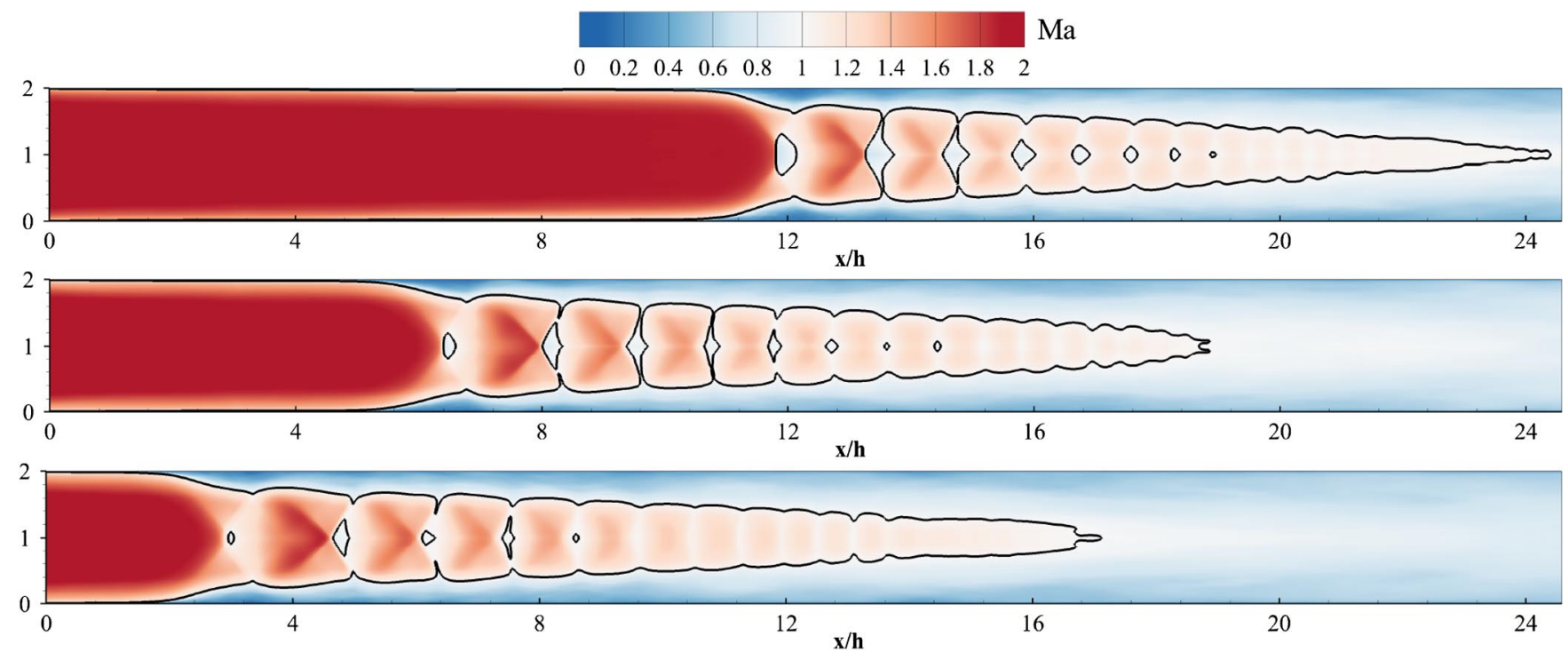

Fig. 8 Time-averaged contours of Mach number with sonic line in black for case A (top), case B (middle), and case C (bottom).

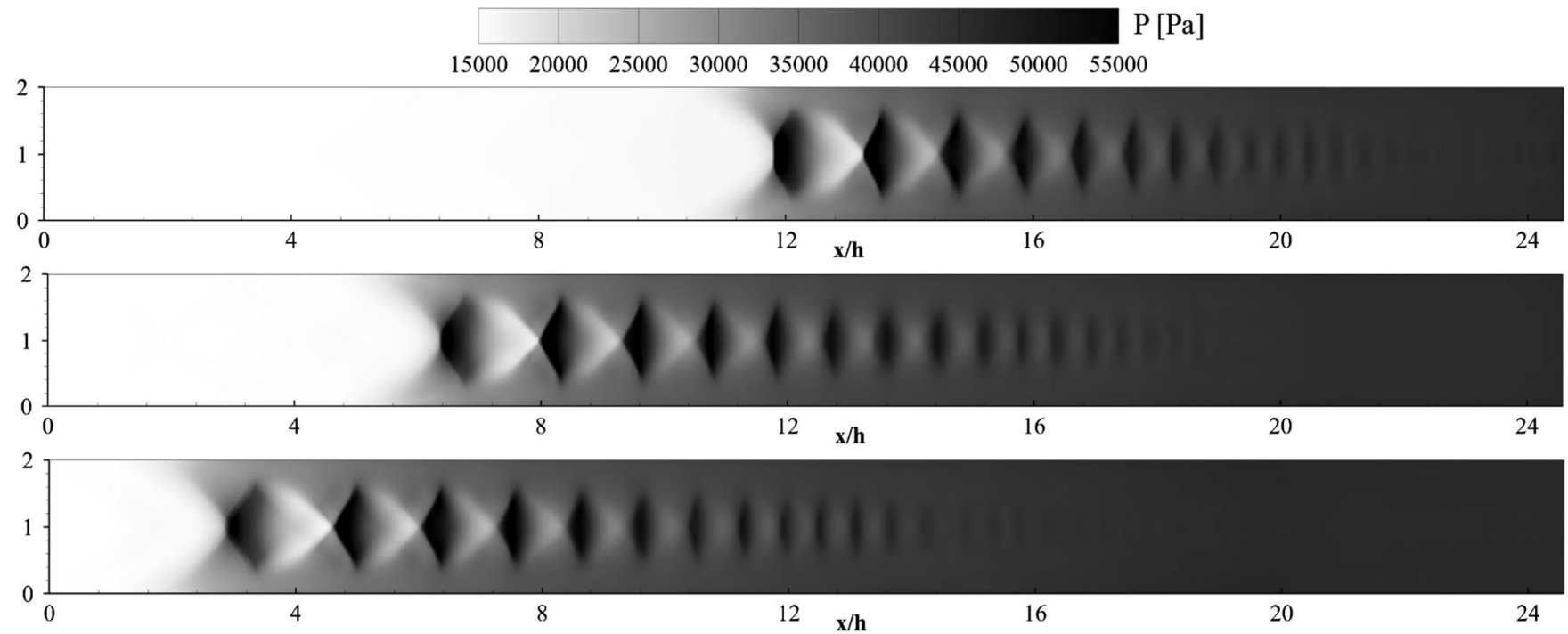

Fig. 9 Time-averaged contours of static pressure for case A (top), case B (middle), and case C (bottom).

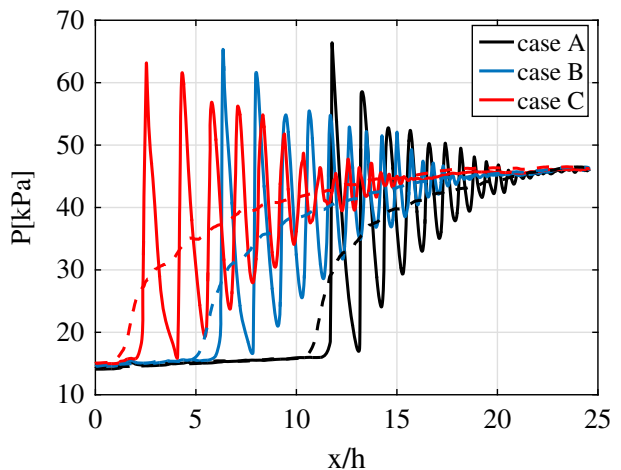

Fig. 10 Centerline (solid) and wall (dashed) static pressure profiles for all cases.

shock-generated detachment decreases. The generation of the next shock structure is again based on the local confinement ratio. As a result, the distance between the shocks depends mainly on the core Mach number. The discrepancies between the cases thus build up after each compression/expansion cycle.

In summary, the inlet boundary-layer thickness determines the normal-like portion of the leading shock. This directly impacts compression efficiency as well as the size and spacing of the diamond cells. Ultimately, the length of the shock train is determined based on the applied backpressure and the boundary-layer thickness.

\section{Effect of Inflow Boundary-Layer Thickness Fluctuations on Shock Trains}

In practical scramjet designs, the downstream combustor section will respond to shock-train movements, typically through a thermal throat located downstream in the combustor. Another way to introduce these motions is through upstream boundary-layer variations, which is considered in this study. As discussed in Sec. I, boundary-layer changes are also useful for active control of the unstart process. Hence, a critical design aspect is the dynamic response of the shock train to backpressure or inflow confinement ratio fluctuations. Although experiments can cover much lower frequencies (less than $10 \mathrm{~Hz}$ [17]), only higher frequencies (greater than $10 \mathrm{~Hz}$ ) are tractable in high-fidelity simulations. To capture the main response characteristics, numerical simulations have to span a computational time of the order of $100 \tau_{c}$, which is out of reach for DNS-like resolutions.

In this section, we propose to investigate the dynamic response of the shock-train structure to inlet boundary-layer height harmonic perturbations. To access the lower range of the accessible 

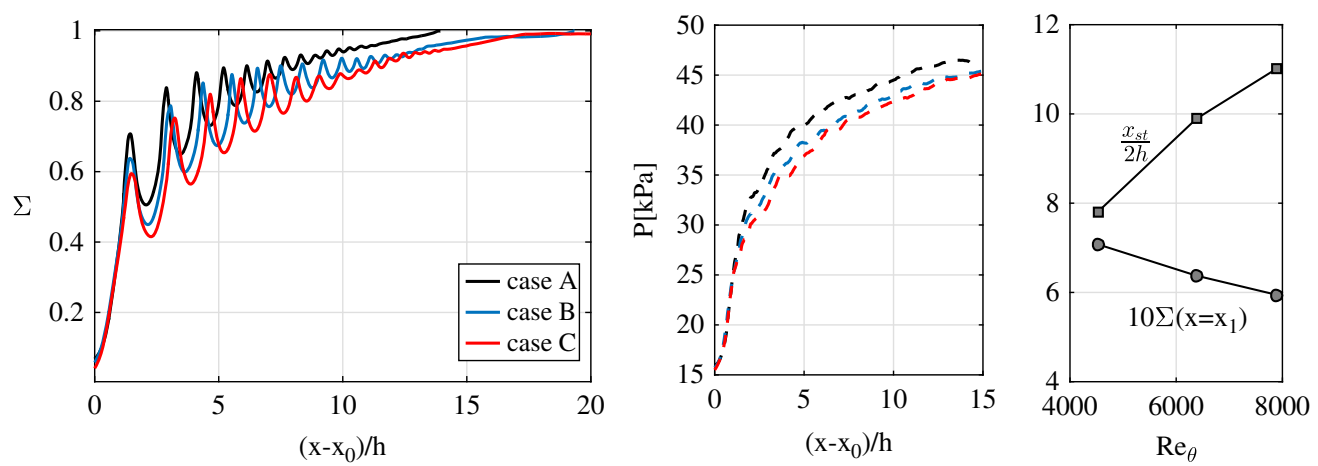

Fig. 11 Integral of the normalized static pressure gradient $\Sigma$ over the isolator rectangular cross section (left). Wall pressure rise profiles along the pseudoshock (middle). Evolution of shock-train length ( $\square)$ and leading compression strength $10 \times \Sigma\left(x_{1}\right)(\bullet)$ as a function of inflow $R e_{\theta}($ right).

frequencies, the simulations are further accelerated by first adopting the coarser grids R1 and later R2 defined in Sec. II. The section will be divided into three parts. First, a broad spectral range of inflow harmonic oscillations will be investigated on grid R1 over large physical time scales on the order of $100 \tau_{c}$. Second, because grid R1 was shown in Sec. II to be underresolving the shock-train structure, one additional simulation will be performed on grid R2 at any particular frequency of interest revealed by the first scan. Last, the shock-train response to inflow oscillations will be described in light of the results obtained from all simulations.

\section{A. Broad Spectral Investigation}

As previously mentioned, the unsteadiness observed in Sec. III is not present on grid R1. This creates a steady shock train for case B inflow conditions (seen in the centerline pressure space-time contour shown in Fig. 12), which serves as a baseline case. Because no oscillations are found in the shock-train length and position, any unsteadiness observed would be the exclusive shock-train response to the inflow oscillations. The wall pressure profiles were found to compare relatively well with the DNS (Fig. 3) and will be used in the forthcoming analysis to investigate the temporal response of the coarse shock train.

The inflow perturbation is introduced by temporally interpolating between the inflow fields for case A and C. Figure 13 shows the

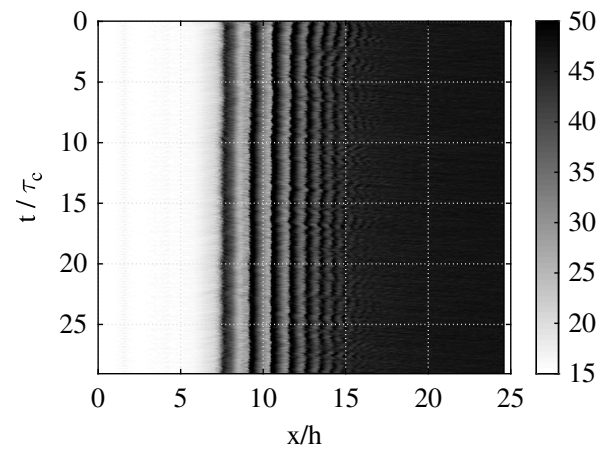

Fig. 12 Space-time plot of the centerline pressure (in kilopascals) for case $B$ when using the coarse grid $R 1$.

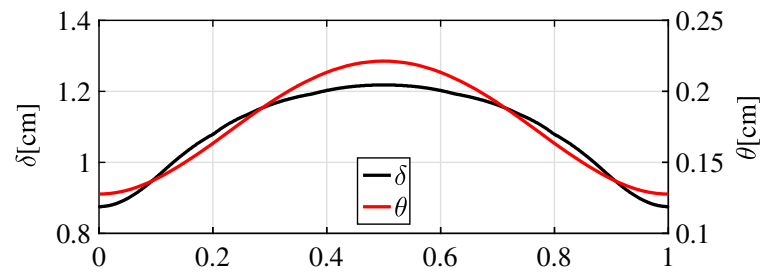

$\mathrm{t} / \mathrm{T}$

Fig. 13 Variation of inflow boundary-layer thickness over one oscillation period. theoretical variation in momentum $\theta$ and boundary-layer $\delta$ thicknesses over one period. Six different cases, corresponding to cycling frequencies $f_{i} \in[20 ; 60 ; 100 ; 200 ; 500 ; 1000 \mathrm{~Hz}]$ were simulated. Each simulation ran on 4000 cores for $120 \mathrm{~h}$, and data were sampled at a $10 \mathrm{kHz}$ rate. In each case, the wall pressure profile is used to estimate the shock-train length. For instance, Fig. 14 shows on the left the instantaneous bottom wall pressure trace as well as a filtered trace. The foot of the shock is estimated based on the earliest location where the filtered pressure rises to $10 \%$ of the difference between the inflow and backpressure. The midpoint of the shock train is determined as $50 \%$ of that pressure difference value, whereas its tail is located at $80 \%$ of the pressure difference. For the forthcoming discussion, the shock-train length is defined as the distance between the 10 and $80 \%$ pressure rise locations. Its unperturbed length is of $7.66 h$.

First, the temporal evolution of the 10,50, and $80 \%$ backpressure rise locations is analyzed. Figure 14 presents these signals for the 20 , 100 , and $500 \mathrm{~Hz}$ cases only (for the sake of brevity). The $20 \mathrm{~Hz}$ case exhibits similar amplitude oscillations for all 10, 50, and 80\% signals, although the shock-train foot and tail are slightly out of phase due to the time taken for the perturbation to propagate downstream. At $500 \mathrm{~Hz}$ frequency, the shock train is essentially unchanged, indicating that this is too high a frequency to be accommodated by the system. However, the $100 \mathrm{~Hz}$ intermediary case presents some interesting nonlinearity because it is not merely a blend of the lowfrequency and high-frequency solutions. The shock-train foot amplitude oscillation does decrease compared to the $20 \mathrm{~Hz}$ case, but the tail response stays as strong and is out of phase. To further understand the frequency response, it is useful to define a normalized shock-train length defined as the distance between the wall 10 and $80 \%$ pressure growth locations. These time-varying locations are called $x_{10}$ and $x_{80}$ :

$$
\chi_{s t}(t)=\frac{\left(x_{80}-x_{10}\right)(t)}{\left(x_{80}-x_{10}\right)(t=0)}
$$

In the forthcoming discussion, $\xi_{s t, i}$ refers to the single-side amplitude spectra of $\chi_{s t}$ when excited with frequency $f_{i}$.

Figure 15 shows $\chi_{s t}(t)$ plotted as a function of normalized time for the different frequency perturbations. The single-sided amplitude spectra $\xi_{s t, i}$ of these signals is shown at the bottom left. Not surprisingly, the most energetic spectral content matches the frequency of excitation in all cases. The peak spectral content for each frequency is shown alongside. $\xi_{s t, i}\left(f=f_{i}\right)$ is plotted as a percentage of the undisturbed shock-train length to show the shock-train length sensitivity to a particular excitation frequency. It is seen that the 60-100 $\mathrm{Hz}$ perturbation range leads to a resonancelike behavior because it causes the largest increase (about 15\%) in shock-train length over the unperturbed shock structure. This highlights the springlike behavior of the shock train in response to upstream instabilities, with a resonance frequency $f_{c} \in[60 ; 100 \mathrm{~Hz}]$.

We observe that the resonance is caused when the shock-train compression/expansion cells adjust to the boundary layer as fast as possible. In the phase where the boundary layer shrinks, the leading 

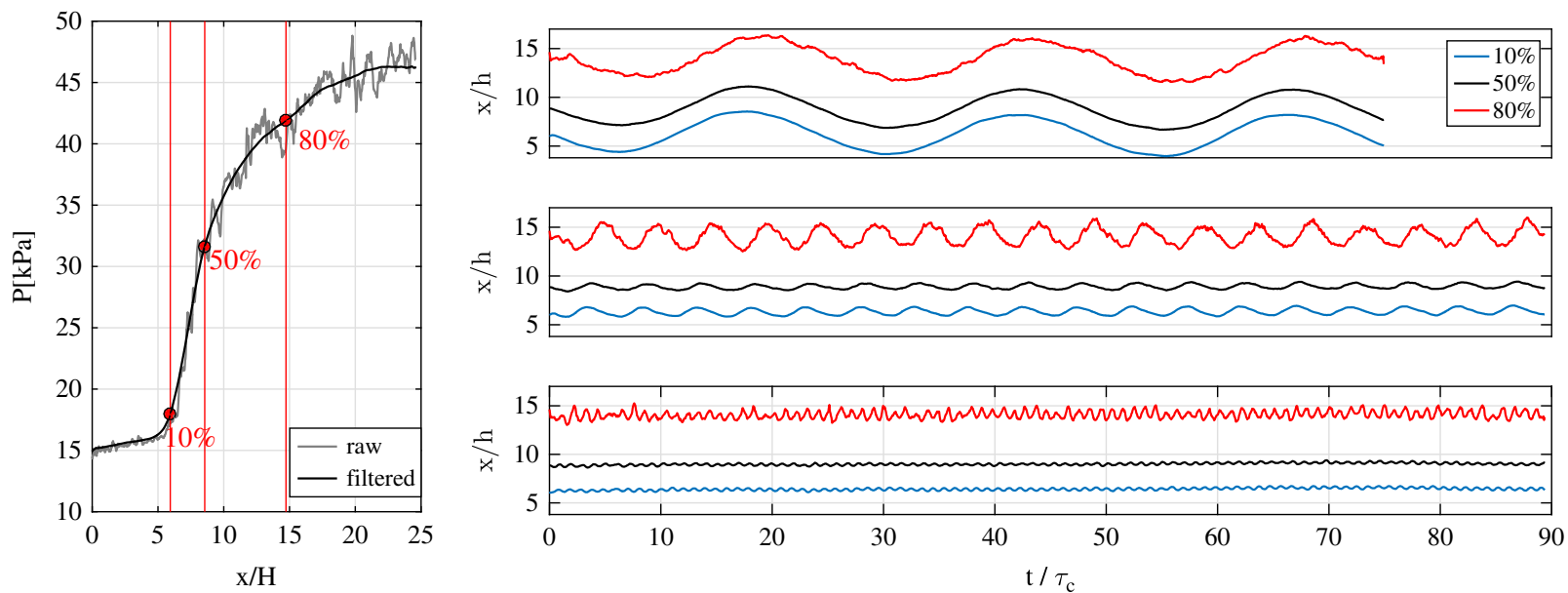

Fig. 14 Locations of 10, 50, and $80 \%$ pressure rise along bottom wall (left). Time variation of these locations (right) for excitation frequencies of (top) $20 \mathrm{~Hz}$, (middle) $100 \mathrm{~Hz}$ and (bottom) $500 \mathrm{~Hz}$.
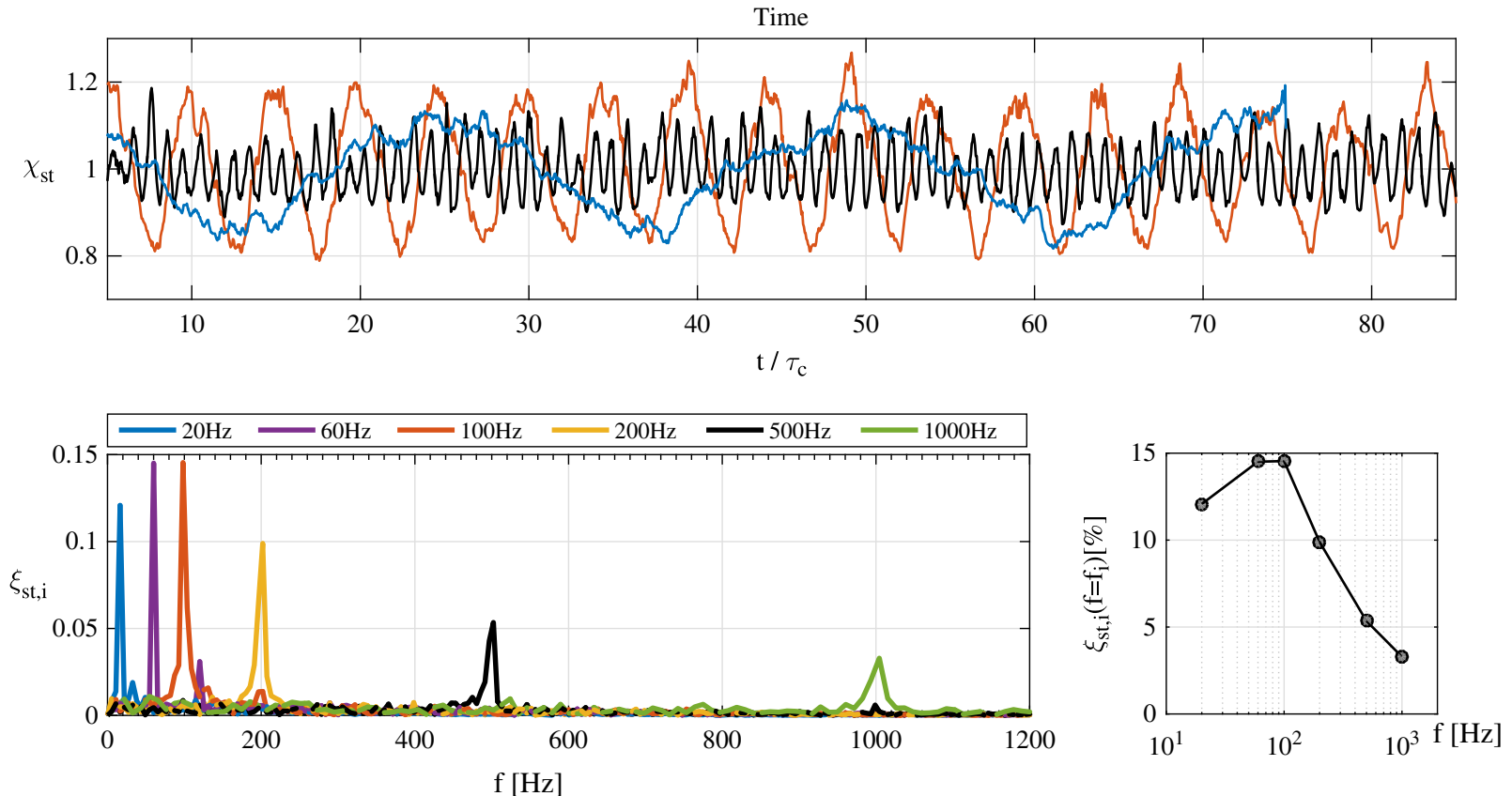

Fig. $15 \chi_{s t}(t)$ for 20,100 and $500 \mathrm{~Hz}$ excitations (top). $\xi_{s t, i}(t)$ for corresponding $f_{i} \in[20 ; 60 ; 100 ; 200 ; 500 ; 1000 \mathrm{~Hz}]$ (bottom left). Amplification of shock train length in percentage of unperturbed length (bottom right).

shock moves downstream, pushing the other cell structures farther downstream, thereby reducing the size of the compression/expansion cell sizes (this corresponds to case A). The opposite is true when the boundary layer grows (corresponding to case C). The cell size adjustment to the inlet boundary layer starts at the foot of the shock train (leading shock) and propagates to the tail (trailing shock), with an observable delay as the shocks interact with their neighbors. This causes a phase lag that is augmented by each compression/expansion cycle. This is the same mechanics illustrated in Fig. 7; moving from the earliest time snapshot, it can be seen that the first shock pushes the next shock, and so on, until the entire shock train has adjusted to the change in inflow condition.

The resonance frequency can be determined with greater accuracy by measuring the phase lag between the time-varying wall pressure locations $x_{10}$ and $x_{80}$. The fluctuating components of these two quantities are $x_{10}^{\prime}$ and $x_{80}^{\prime}$, respectively, and are defined as the fluctuation about the arithmetic average of $\overline{x_{10}}$ and $\overline{x_{80}}$ from $x_{10}$ and $x_{80}$. Figure 16 shows the time variation of these two fluctuating quantities. In addition, the cross-correlation between the two signals is also shown. This quantity corresponds to the convolution product $x_{10}^{\prime} \star x_{80}^{\prime}(\tau)$, where $\tau$ is the time separation of the signals. The red line gives the location of maximum correlation, whereas the dashed black line locates the origin. Note that the maximum correlation location does not coincide with zero time difference, indicating that there is a time lag between the oscillations at the two locations. This time lag is indicated in the top-right corner of all $x_{10}^{\prime} \star x_{80}^{\prime}$ plots. Using the corresponding excitation frequency, the time lag is converted to a phase lag. The measured phase lag between the $x_{10}^{\prime}$ and $x_{80}^{\prime}$ signals for the $20,60,100$, and $200 \mathrm{~Hz}$ oscillation cases were $23.7,64.8,100.8$, and $129.6 \mathrm{deg}$, respectively. Resonance happens for a phase lag of $90 \mathrm{deg}$, and the resonance frequency $f_{c}$ is estimated at $93 \mathrm{~Hz}$ using linear interpolation for this shock train. This is within the peak range of spectral response based on the experiment, as mentioned at the end of Sec. II. Figure 16 also reveals the strength of the resonance. For comparison, Fig. $\overline{15}$ shows a $12 \%$ increase of shock-train length at $20 \mathrm{~Hz}$ and a modest $15 \%$ increase of shock-train length at $100 \mathrm{~Hz}$, which is close to the resonance frequency. From Fig. 16, the oscillation amplitude for $x_{10}^{\prime}$ is much smaller at $100 \mathrm{~Hz}(\approx \overline{0.37} \mathrm{~h})$ than at $20 \mathrm{~Hz}(\approx 2.01 \mathrm{~h})$. Hence, the $15 \%$ increase in shock-train length corresponds to a much higher amplitude ratio between the tail and foot at $100 \mathrm{~Hz}(2.64)$ than at $20 \mathrm{~Hz}(1.01)$. Had the $x_{10}^{\prime}$ amplitude oscillation remained constant for all excitation frequencies, the 

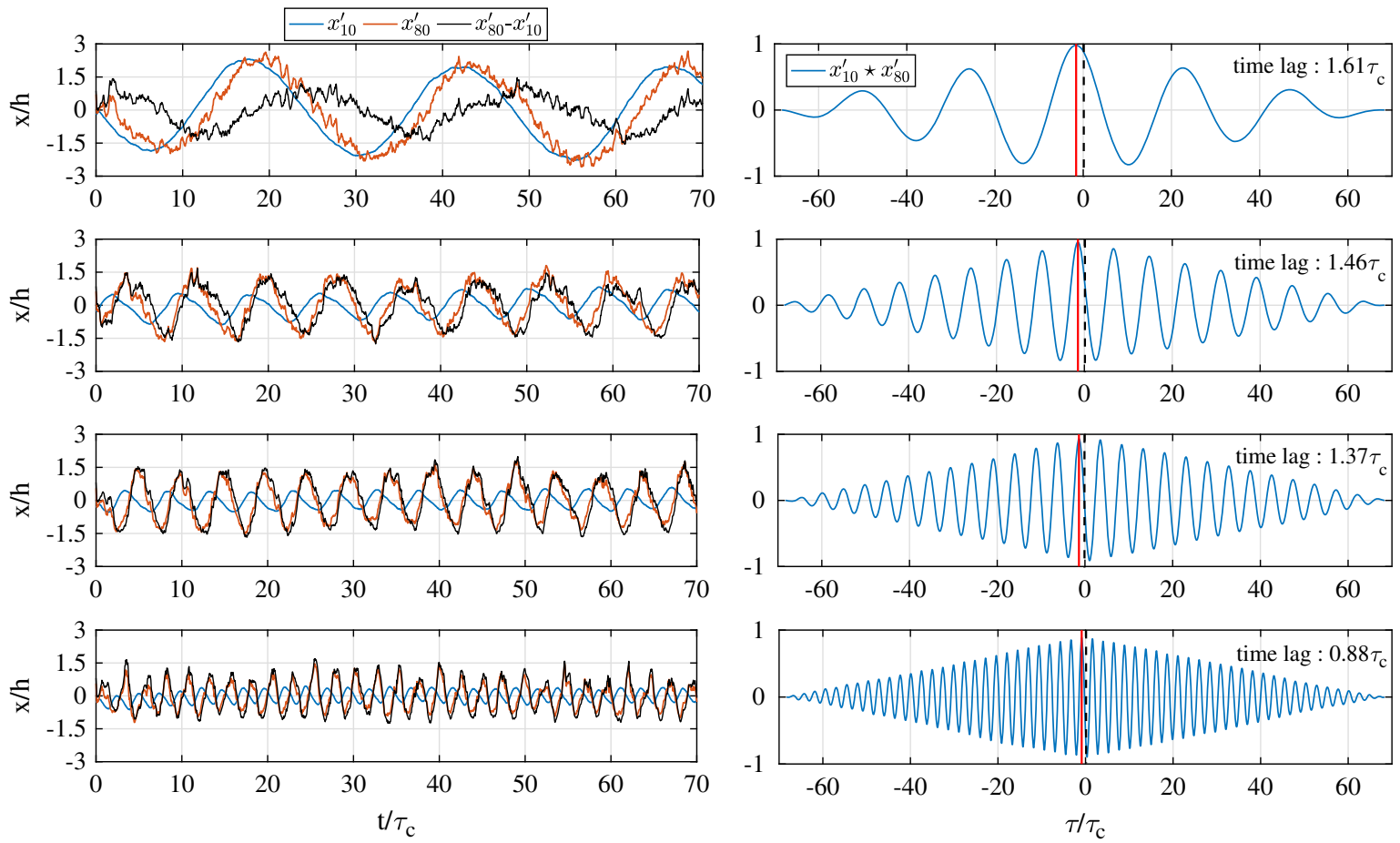

Fig. $16 x_{10}^{\prime}, x_{80}^{\prime}$, and $x_{80}^{\prime}-x_{10}^{\prime}$ (from top to bottom) for 20, 60, 100 and $200 \mathrm{~Hz}$ excitation (left). Corresponding cross-correlation $x_{10}^{\prime} \star x_{80}^{\prime}$, red line indicates the peak location (right).

oscillation of the shock-train length at $100 \mathrm{~Hz}$ could have been as high as $2.01 / 0.37 \times 15 \%=81 \%$ of the unperturbed length. Such resonance could potentially disrupt the shock-train compression ratio and even move it out of the isolator, provoking the unstart of the engine. More interestingly, this analysis reveals that a low-pass filter precedes the springlike behavior of the isolator, which reduces the oscillation amplitude of the shock train. This aspect is further investigated later.

The $x_{10}^{\prime}$ and $x_{10}^{\prime}$ signals spectral contents $\xi_{10, i}$ and $\xi_{80, i}$ are now separately analyzed using the same process as $\chi_{s t}$. Figure 17 is the equivalent of Fig. 15 and quantifies the oscillation amplitudes normalized by the initial shock-train length of both the foot and tail of the shock train as a function of the excitation frequency. Although the oscillation at the lowest frequency is the largest (about 25\% of the unperturbed shock-train length), it is remarkably equal for both foot and tail. This suggests that there is little interest investigating frequencies lower than $20 \mathrm{~Hz}$ because the shock train's dynamic response seems to fully accommodate such high wavelengths (response time much smaller than the excitation). The foot and tail oscillation amplitude might increase but remain equal, and the shock train would just translate inside the isolator in between the solution of cases $\mathrm{A}$ and $\mathrm{C}$. As the frequency increases, the reduction in oscillations of the shock train is more pronounced at the foot than the tail of the shock train, which is also observable in Fig. 16. The

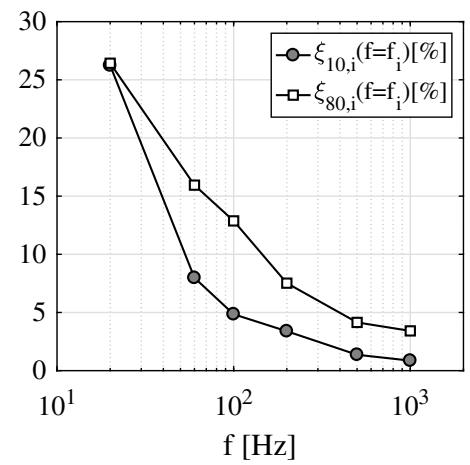

Fig. 17 Amplitude variation of $x_{10}$ and $x_{80}$ for all excitation frequencies $f_{i}$ expressed as percent of the unperturbed shock-train length. boundary layer detaches at the shock-train foot, and the $10 \%$ pressure rise location resides in the recirculation bubble slightly upstream of the axial position of the first shock, as seen in Fig. 10. Because this separation bubble has very low dynamical response due to the highly viscous and low-speed fluid contained within, the response of the shock-train also is damped with increasing perturbation frequency. Further, the static pressure in the recirculation bubble tends to be more uniform (that is, spatially homogenized) and would therefore be less sensitive to high-frequency changes to the flowfield. On the contrary, the $80 \%$ pressure rise location is directly located under the trailing shocks in the shock train and is more directly correlated to their strength.

As noted earlier, the grid coarsening introduces artificial numerical dissipation, which dissipates the highest-frequency structures in the flow. Increased resolution will alter shock-train response by changing the dynamics of the recirculation bubble at the shock foot. Further, it was also observed that the R1-grid-based shock-train structure differs from the R2 and R3 profiles, especially weakening the centerline compression and expansion waves. Because resonance seems to be partly triggered by the phase lag between the leading and tailing shocks, its characteristic frequency would logically be affected by an increase in strength and number of shocks. Hence, it is entirely plausible that a resonance will be observed on finer grids as well, albeit with different transfer functions than in Fig. 15. Finally, the large-amplitude ratio between the tail and foot oscillation (Fig. 16) is the second cause for the increase in shock-train length. Its causes are not elucidated yet, and it remains to be seen whether it would happen on higher-resolution grids.

\section{B. Grid-Resolved Oscillation Analysis}

To address these concerns, a last simulation is run on grid $\mathrm{R} 2$ at the resonance frequency of $93 \mathrm{~Hz}$ estimated from R1 calculations. Because the R2 simulations presented in Sec. II are slowly convecting upstream, the backpressure is reduced from $\overline{70}$ to $65 \%$ normal shock rise to stabilize the shock train and avoid a contamination of the forced inflow oscillation with large-scale motions. This simulation has run on 8000 cores for 360 wall-clock hours. This represents a physical runtime of $68.4 \mathrm{~ms}$ or $33.4 \tau_{c}$, corresponding to 6.4 oscillation periods. The shock train accommodates the lower backpressure and becomes stable during the first $10 \tau_{c}$. The 

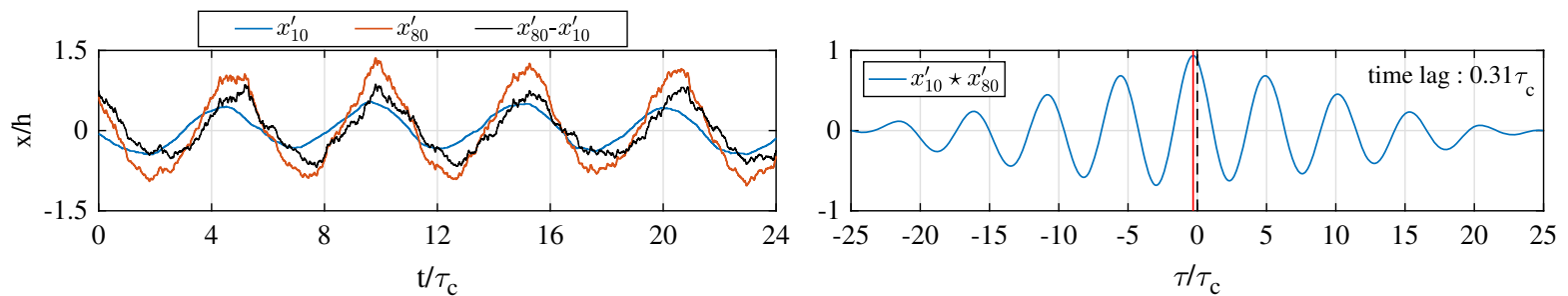

Fig. $18 x_{10}^{\prime}, x_{80}^{\prime}$, and $x_{80}^{\prime}-x_{10}^{\prime}$ for $93 \mathrm{~Hz}$ excitation on grid R2 (left). Corresponding cross-correlation $x_{10}^{\prime} \star x_{80}^{\prime}$, red line indicates the peak location (right).

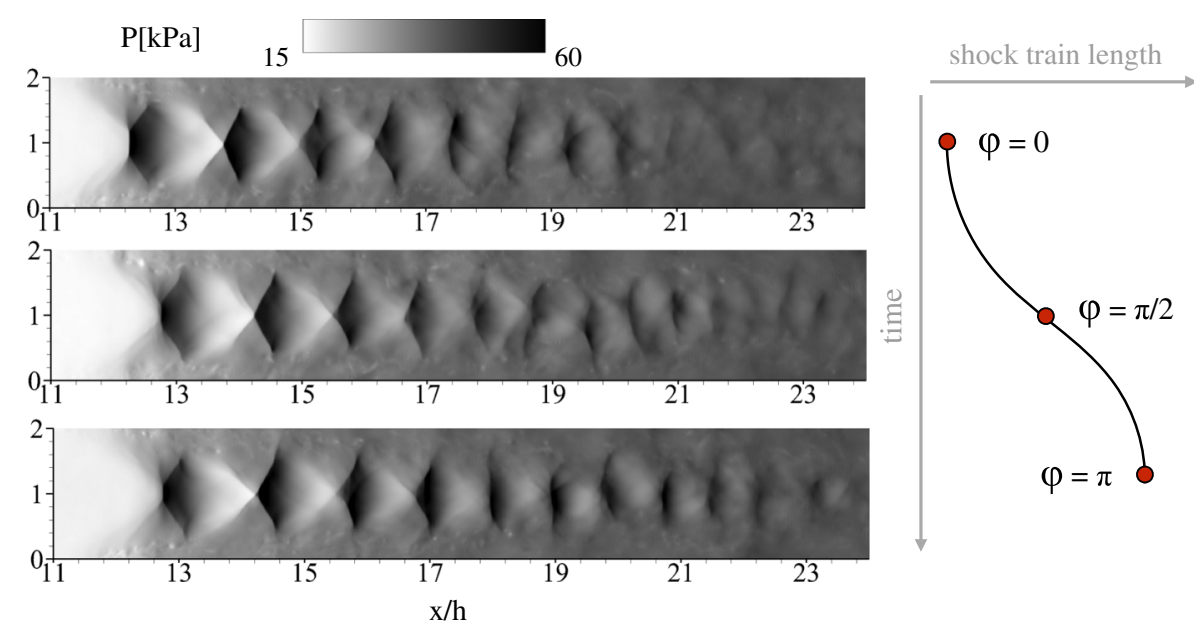

Fig. 19 Snapshots of static pressure contours at various times over one half-oscillation period.

forthcoming analysis is based on data collected after this initial period. Additionally, the sampling rate is increased from 10 to $476 \mathrm{kHz}$ to permit time-resolved analysis on the high-speed centerline flow.

The R2 grid simulation is first used to analyze wall pressure similarly to the $\mathrm{R} 1$ grid results shown in Fig. 18 . The time lag between the $x_{10}^{\prime}$ and $x_{80}^{\prime}$ signals is measured at $0.31 \overline{\tau_{c}}$, giving an equivalent phase lag of $21 \mathrm{deg}$. This is less than the measured value on the coarse grid; a higher oscillation frequency would be necessary to trigger a 90 deg phase lag. Because the resolution of grid R2 is twice higher than grid R1, the cutoff frequency is also doubled. Because the phase lag decreases by a factor $\approx 4$, we can deduce that the smallest length scales (located in the boundary layer) play a paramount role in shock-train dynamic. Consequently, the characteristic frequency is underestimated when low wavelengths are filtered out. Yet the amplitude of shock-train length oscillations is similar to the R1 simulations given the change of backpressure and resolution. A $\xi_{s t}(f=93 \mathrm{~Hz})$ of $11.1 \%$ is computed, with a $\xi_{10}(f=93 \mathrm{~Hz})$ and $\xi_{80}(f=93 \mathrm{~Hz})$ of 7.4 and $17.6 \%$, respectively. On grid $\mathrm{R} 1$, these values are respectively of $14.5,5.4$, and $13.4 \%$ (using a linear interpolation at $93 \mathrm{~Hz}$ on Figs. 15 and 17).

As seen in Fig. 19, the change in shock-train length over half a period (phase angle $\phi=[0, \pi / 2, \pi]]$ ) is very noticeable, particularly in terms of the number of shocks. Therefore, the refined grid simulation shows an increase of shock-train length primarily due to the ratio between its tail and foot oscillation amplitudes, rather than a consequent phase lag (which is still present). This suggests that a model of the isolator response mechanism solely based on the phaselag resonance phenomenon (analogous to a conventional harmonic oscillator) is not complete.

In an effort to identify the causes of the large oscillation amplitude of the shock-train tail as compared to the foot, the time-resolved centerline pressure signal is scrutinized. Contrary to wall pressure profiles, the centerline profile is not filtered in space to retain the shock resolution but is filtered in time using a moving average filter (a simple unweighted mean) of bandwidth $0.1 \mathrm{~ms}$ (i.e., less than $1 \%$ of an oscillation period). The space-time contour of the shock-train length is shown in Fig. 20. It is seen that the oscillation waveforms

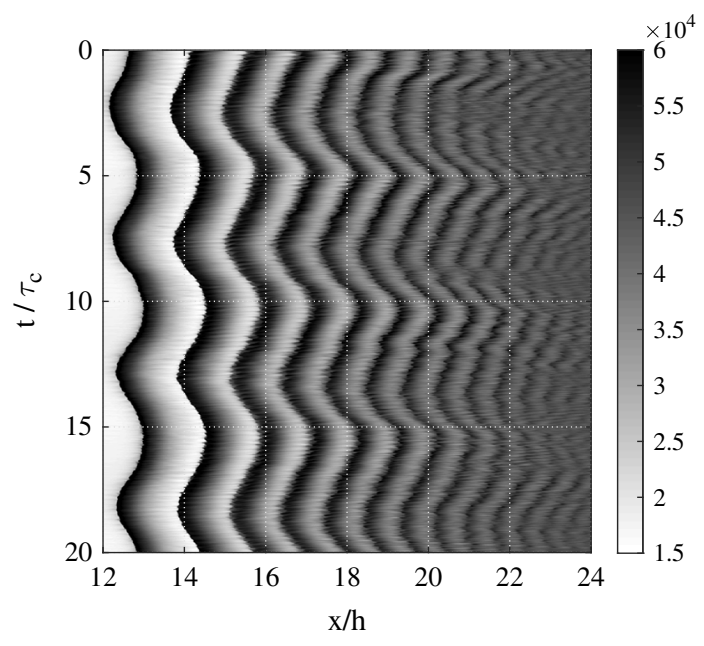

Fig. 20 Space-time plot of the centerline pressure (in kilopascals) for the $93 \mathrm{~Hz}$ oscillation case on grid R2.

tend to distort from a clean sinusoidal form at downstream locations. Further, the shock-train tail fades away when the structure is convecting downstream.

The time-varying locations and amplitudes of the constituent shocks are extracted. In Fig. 21, the phase angle $\phi$ based on the $93 \mathrm{~Hz}$ frequency oscillation is plotted as a function of two shock-related quantities: 1) the pressure $\tilde{P}_{s}$, defined as the peak pressure of shock number $s$ normalized by the backpressure, and 2) the velocity $\tilde{u}_{s}$, which is defined as the local convective velocity of the $s$ th shock normalized by the inlet bulk speed. The vertical dashed line represents $\tilde{u}_{s}=0$, whereas the horizontal line gives the average of all $\tilde{P}_{s}$ realizations. A $5 \%$ amplitude of $\tilde{u}_{s}$ (half the range of the plots) equals to a velocity variation of $\approx 26 \mathrm{~m} / \mathrm{s}$. Because the velocity decreases with downstream distance, this $5 \%$ amplitude corresponds to an increasing fraction of the local flow velocity. As a result, the amplitude of the sth shock pressure oscillation increases as $s$ 


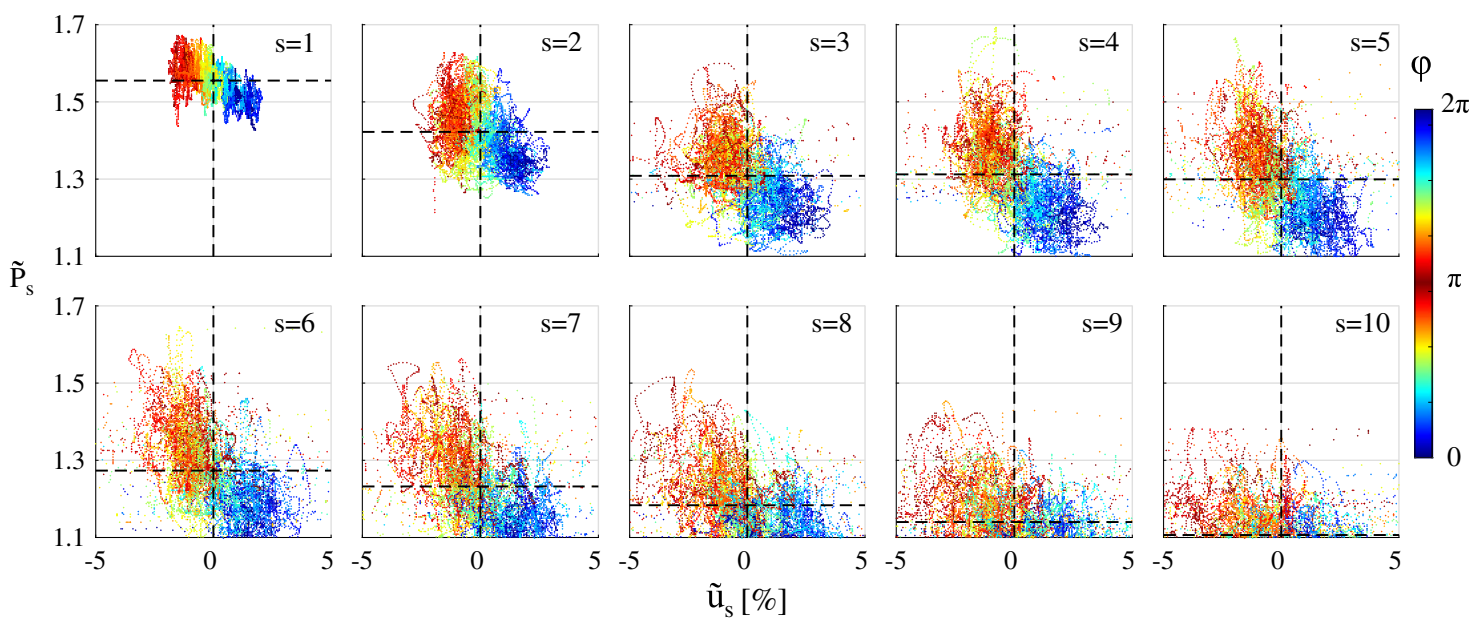

Fig. 21 Correlations of convective velocity $u_{s}$ with compression rate $\tilde{P}_{s}$ for the first 10 shocks of the shock train.

increases; this shows up as an increased scattering in the $P_{s}-u_{s}$ plot. This increases the sensitivity of the tail to large-scale oscillations, which alternatively force the shock train into a weak or strong state, as identified in Fig. 7. The apparent Mach number range values defined in Sec. III.B increase with the convective velocity, such that the last shocks vanish $(M a=<1)$ in the weak state. The tail of the shock train weakens and disappears at $\phi=0$, then strengthens and reappears at $\phi=\pi$. This phenomenon is postulated to be the cause of the large ratio between $x_{10}^{\prime}$ and $x_{80}^{\prime}$ observed in all simulations for frequencies superior to $20 \mathrm{~Hz}$.

\section{Mechanics of Shock-Train Length Fluctuations}

From the discussion in the previous sections, it is possible to derive three different physical processes that affect shock-train length when the inflow conditions are perturbed.

1) Shock-to-shock time-lagged interactions: here, the upstream perturbation propagates through the cells with a certain time-lag depending on a local wave-speed. The wave speed depends on both the perturbation frequency and size of the compression-expansion cells. Because the large scales drive this mechanism, even coarse grids could capture this process. When the phase lag is $90 \mathrm{deg}$, resonance behavior similar to harmonic oscillators is observed.

2) Apparent shock Mach number: in addition to the harmonic oscillator behavior, the length of the shock train also depends on its convective velocity relative to the flow velocity. To achieve a shock convective velocity on the order of the bulk streamwise velocity $U$ and appreciably affect its apparent Mach number, the oscillation frequency $f_{s}$ and amplitude $A_{s}$ must be high enough such that $U \approx A_{s} f_{s}$. This effect introduced another characteristic frequency, which converts the isolator into an anharmonic oscillator. If the frequency $f_{s}$ is high enough, the shock-train tail will periodically disappear, which will change its length even if the shock-to-shock phase lag is far from 90 deg. This is observed in the R2 grid simulations (Sec. IV.B).

3) Leading shock shape: as the inflow boundary-layer thickness is varied, the shock train moves up- or downstream. In addition, the leading shock undergoes changes in shape. When the leading shock is formed from a thin boundary layer, the normal portion of the lambdashaped shock structure grows, increasing the pressure jump across this shock and decreasing the overall shock-train length. When a thicker boundary layer is encountered, the oblique part of the shock dominates, causing a growth in the length but a decrease in the pressure jump across the leading shock. From the data collected, it is not clear how fast the shock structures adapt to the changing boundary layer. Hence, the exact impact of this change in shock structure in a dynamic environment is not fully understood yet.

Another observation related to the mechanism is the importance of the transverse variations in the flowfield. The momentum thickness variation amplitude is $0.027 \mathrm{~h}(0.45 \mathrm{~mm})$ and is applied in the transverse direction. Yet the shock-train motion can be roughly two orders of magnitude larger in the axial direction, whereas its length oscillates at a maximum amplitude of $40 \mathrm{~mm}$. The resonance phenomenon is greatly attenuated by the upstream low-pass response of the shock-train foot. Hence, even minor changes to the inflow boundary layer can have a significant impact on the shocktrain stability should the low-pass region be too small to filter these out. Also, the artificial increase of viscosity $(x 4)$ increases the wall shear stress and contributes to the damping of the recirculation bubble displacement. Finally, only sinusoidal excitations have been considered in this study. It may be the case that the shock train is relatively robust to broadband fluctuations in the inflow boundary layer.

\section{Conclusions}

Direct numerical simulations of shock trains in a turbulent channel have been carried out. The database contains shock-train data at different inflow conditions, characterized by variations in boundarylayer thickness. The simulations show that, when the boundary-layer thickness is small, the shock train is pushed toward the exit. Additionally, the normal-like portion of the leading bifurcating shock increases with small inflow confinement ratio, leading to shorter but stronger compression/expansion diamond cells. When the inflow conditions are varied over time, the shock-train response is nontrivial and depends on the excitation frequency. With too high or too low an excitation frequency, the shock train either stays nearly stationary or moves coincidentally to the inflow variation. At higher frequencies, two additional physical processes contribute to the appearance of a resonance phenomenon (increase of shock-train length). First, the perturbations travel downstream at finite speed, and a phase lag between the train's foot and tail appears, similar to a harmonic oscillator. Second, the shock's strength is correlated to their convective velocity relative to the flow stream. Shocks at the tail of the train therefore weaken and disappear while convecting downstream or strengthen and reappear while convecting upstream, further increasing the shock-train length oscillation amplitude. These two processes are triggered at different characteristic frequencies, defining the shock train as an anharmonic oscillator. Additionally, it is deduced that the low-pass filter nature of the leading shock prevents the shock train from exhibiting much larger spatial oscillations. Nevertheless, even small variations in the inflow boundary-layer thickness are seen to cause large changes in shock-train length.

\section{Acknowledgments}

This work was financially supported through a U.S. Air Force Research Laboratory grant FA9550-12-1-0460 with Ivett Leyva as the Program Manager. We would like to thank Mirko Gamba and Robin Klomparens for sharing their experimental data before publication. The authors also gratefully acknowledge the generous allocation of computing time by the NASA High-End Computing Program through the NASA Advanced Supercomputing Division at Ames Research Center. 


\section{References}

[1] Matsuo, K., Miyazato, Y., and Kim, H.-D., "Shock Train and PseudoShock Phenomena in Internal Gas Flows," Progress in Aerospace Sciences, Vol. 35, No. 1, 1999, pp. 33-100. doi:10.1016/S0376-0421(98)00011-6

[2] Carrol, B., and Dutton, J., "Characteristics of Multiple Shock-Wave Turbulent Boundary-Layer Interactions in Rectangular Ducts," Journal of Propulsion and Power, Vol. 6, No. 2, March-April 1990, pp. 186-193.

doi: $10.2514 / 3.23243$

[3] Sullins, G., and Mclafferty, G., "Experimental Results of Shock Trains in Rectangular Ducts," 4th Symposium on Multidisciplinary Analysis and Optimization, AIAA Paper 1992-5103, 1992.

[4] Waltrup, P. J., and Billig, F. S., "Structure of Shock Waves in Cylindrical Ducts," AIAA Journal, Vol. 11, No. 10, 1973, pp. 1404-1408. doi: $10.2514 / 3.50600$

[5] Carrol, B., and Dutton, J., "Multiple Normal Shock-Wave Turbulent Boundary-Layer Interactions," Journal of Propulsion and Power, Vol. 8, No. 2, March-April 1992, pp. 441-448. doi: $10.2514 / 3.23497$

[6] Wagner, J. L., Yuceil, K. B., and Clemens, N. T., "Velocimetry Measurements of Unstart of an Inlet/Isolator Model in a Mach 5 Flow," AIAA Journal, Vol. 48, No. 9, 2010, pp. 1875-1888. doi:10.2514/1.J050037

[7] Sugiyama, H., Tsujiguchi, Y., and Honma, T., "Structure and Oscillation Phenomena of Pseudo-Shock Waves in a Straight Square Duct at Mach 2 and 4," 15th AIAA International Space Planes and Hypersonic Systems and Technologies Conference, AIAA Paper 2008-2646, April-May 2008.

[8] Arai, T., Sugiyama, H., Abe, F., Takahashi, T., and Onodera, O., "Internal Structure of Pseudo-Shock-Waves in a Square Duct," Current Topics in Shock Waves: 17th International Symposium on Shock Waves and Shock Tubes, edited by Y. W. Kim, Vol. 208, AIP Conference Proceedings, American Inst. of Physics (AIP) Publ., Melville, NY, 1990, pp. 850-855.

[9] Do, H., Im, S.-K., Mungal, M. G., and Cappelli, M. A., "The Influence of Boundary Layers on Supersonic Inlet Flow Unstart Induced by Mass Injection," Experiments in Fluids, Vol. 51, No. 3, Sept. 2011, pp. 679-691. doi:10.1007/s00348-011-1077-3

[10] Ikui, T., Matsuo, K., Nagai, M., and Honjo, M., "Oscillation Phenomena of Pseudo-Shock Waves," Bulletin of the JSME-Japan Society of Mechanical Engineers, Vol. 17, No. 112, 1974, pp. 1278-1285.

[11] Tan, H. J., Sun, S., and Huang, H. X., "Behavior of Shock Trains in a Hypersonic Inlet/Isolator Model with Complex Background Waves," Experiments in Fluids, Vol. 53, No. 6, Dec. 2012, pp. 1647-1661. doi:10.1007/s00348-012-1386-1

[12] Valdivia, A., Yuceil, K. B., Wagner, J. L., Clemens, N. T., and Dolling, D. S., "Control of Supersonic Inlet-Isolator Unstart Using Active and Passive Vortex Generators," AIAA Journal, Vol. 52, No. 6, June 2014, pp. 1207-1218. doi:10.2514/1.J052214

[13] Handa, T., Masuda, M., and Matsuo, K., "Three-Dimensional Normal Shock-Wave/Boundary-Layer Interaction in a Rectangular Duct," AIAA Journal, Vol. 43, No. 10, Oct. 2005, pp. 2182-2187. doi: $10.2514 / 1.12976$

[14] Weiss, A., and Olivier, H., "Behaviour of a Shock Train Under the Influence of Boundary-Layer Suction by a Normal Slot," Experiments in Fluids, Vol. 52, No. 2, Feb. 2012, pp. 273-287. doi:10.1007/s00348-011-1211-2

[15] Geerts, J. S., and Yu, K. H., "Shock Train/Boundary-Layer Interaction in Rectangular Isolators," AIAA Journal, Vol. 54, No. 11, 2016, pp. 3450-3464.

[16] Klomparens, R. L., Driscoll, J. F., and Gamba, M., "Unsteadiness Characteristics and Pressure Distribution of an Oblique Shock Train," 53rd AIAA Aerospace Sciences Meeting, AIAA Paper 2015-1519, 2015. doi:10.2514/6.2015-1519

[17] Klomparens, R. L., Driscoll, J. F., and Gamba, M., "Response of a Shock Train to Downstream Back Pressure Forcing," 54th AIAA Aerospace Sciences Meeting, AIAA Paper 2016-0078, 2016.

[18] Bruce, P. J. K., and Babinsky, H., "Unsteady Shock Wave Dynamics," Journal of Fluid Mechanics, Vol. 603, May 2008, pp. 463-473. doi:10.1017/S0022112008001195

[19] Hutchins, K. E., Akella, M. R., Clemens, N. T., Donbar, J. M., and Gogineni, S., "Experimental Identification of Transient Dynamics for Supersonic Inlet Unstart," Journal of Propulsion and Power, Vol. 30, No. 6, Nov.-Dec. 2014, pp. 1605-1612.

doi: $10.2514 / 1 . B 35230$
[20] Campo, L. M., and Eaton, J. K., "Shock Boundary Layer Interactions in a Low Aspect Ratio Duct," International Journal of Heat and Fluid Flow, Vol. 51, Oct. 2014, pp. 353-371. doi:10.1016/j.ijheatfluidflow.2014.10.003

[21] Bolton, J. T., Thurow, B. S., Alvi, F. S., and Arora, N., "Volumetric Measurement of a Shock Wave-Turbulent Boundary Layer Interaction Using Plenoptic Particle Image Velocimetry," 32nd AIAA Aerodynamic Measurement Technology and Ground Testing Conference, AIAA Paper 2016-4029, June 2016.

[22] Koo, H., and Raman, V., "Large-Eddy Simulation of a Supersonic InletIsolator," AIAA Journal, Vol. 50, No. 7, 2012, pp. 1596-1613. doi:10.2514/1.J051568

[23] Fiévet, R., Koo, H., and Raman, V., "Numerical Simulation of a Scramjet Isolator with Thermodynamic Nonequilibrium," 22nd AIAA Computational Fluid Dynamics Conference, AIAA Paper 2015-3418, June 2015.

[24] Su, W.-Y., Ji, Y.-X., and Chen, Y., "Effects of Dynamic Backpressure on Pseudoshock Oscillations in Scramjet Inlet-Isolator," Journal of Propulsion and Power, Vol. 32, No. 2, 2016, pp. 516-528.

[25] Lin, P., Rao, G., and O'Connor, G., "Numerical Analysis of Normal Shock Train in a Constant Area Isolator," 27th Joint Propulsion Conference, AIAA Paper 1991-2162, 1991.

[26] Carrol, B., Lopezfernandez, P., and Dutton, J., "Computations and Experiments for a Multiple Normal-Shock Boundary-Layer Interaction," Journal of Propulsion and Power, Vol. 9, No. 3, MayJune 1993, pp. 405-411. doi: $10.2514 / 3.23636$

[27] Vane,Z., Bermejo-Moreno, I., and Lele, S. K., "Simulations of a Normal Shock Train in a Constant Area Duct Using Wall-Modeled LES," 43rd Fluid Dynamics Conference, AIAA Paper 2013-3204, June 2013.

[28] Morgan, B., Duraisamy, K., and Lele, S. K., "Large-Eddy Simulations of a Normal Shock Train in a Constant-Area Isolator," AIAA Journal, Vol. 52, No. 3, 2014, pp. 539-558. doi:10.2514/1.J052348

[29] Cocks, P. A. T., Bruno, C., Donohue, J. M., and Haas, M., "IDDES of a Dual-Mode Ethylene Fueled Cavity Flameholder with an Isolator Shock Train," 51st AIAA Aerospace Sciences Meeting, AIAA Paper 20130116, Jan. 2013.

[30] Lindstrom, C. D., Davis, D., Williams, S., and Tam, C.-J., "Shock-Train Structure Resolved with Absorption Spectroscopy Part 2: Analysis and CFD Comparison," AIAA Journal, Vol. 47, No. 10, Oct. 2009, pp. 2379-2390. doi: $10.2514 / 1.41077$

[31] Kamali, R., Mousavi, S. M., and Binesh, A. R., "Three Dimensional CFD Investigation of Shock Train Structure in a Supersonic Nozzle," Acta Astonautica, Vol. 116, Nov.-Dec. 2015, pp. 56-67. doi:10.1016/j.actaastro.2015.06.024

[32] Fulton, J. A., Edwards, J. R., Hassan, H. A., McDaniel, J. C., Goyne, C. P., Rockwell, R. D., Cutler, A. D., Johansen, C. T., and Danehy, P. M., "Large-Eddy/Reynolds-Averaged Navier-Stokes Simulations of Reactive Flow in Dual-Mode Scramjet Combustor," Journal of Propulsion and Power, Vol. 30, No. 3, May-June 2014, pp. 558-575. doi:10.2514/1.B34929

[33] Edwards, J. R., and Fulton, J. E., "Development of a RANS and LES/ RANS Flow Solver for High-Speed Engine Flowpath Simulations," 20th AIAA International Space Planes and Hypersonic Systems and Technologies Conference, AIAA Paper 2007-3570, 2015.

[34] Roussel, C., Alizard, F., and Grasso, F., "Turbulence Generation and Sensitivity to Mean Inflow Conditions for a Supersonic Flow in a Rectangular Duct at $M=1.61, " 22 n d$ AIAA Computational Fluid Dynamics Conference, AIAA Paper 2015-2618, June 2015. doi:10.2514/6.2015-2618

[35] Pizzella, M., Warning, S., Jennerjohn, M., McQuilling, M., Purkey, A., Scharnhorst, R., and Mani, M., "Numerical Investigation of a Normal Shock Wave Boundary Layer Interaction in a 4.3 Aspect Ratio Test Section," 54th AIAA Aerospace Sciences Meeting, AIAA Paper 20160614, Jan. 2016.

[36] Riley, L. P., Hagenmaier, M. A., Donbar, J. M., and Gaitonde, D. V., "A Computational Investigation of Unstart in a Dual-Mode Scramjet," 54th AIAA Aerospace Sciences Meeting, AIAA Paper 2016-1901, Jan. 2016.

[37] Ramprakash, A., and Murganandam, T. M., "Experimental Study on Start/Unstart Behavior of Two Dimensional Mixed Compression Inlet by Cowl Actuation," 52nd AIAA/SAE/ASEE Joint Propulsion Conference, AIAA Paper 2016-5072, July 2016.

[38] Bermejo-Moreno, I., Campo, L., Larsson, J., Bodart, J., Helmer, D., and Eaton, J. K., "Confinement Effects in Shock Wave/Turbulent Boundary Layer Interactions Through Wall-Modelled Large-Eddy Simulations," 
Journal of Fluid Mechanics, Vol. 758, Nov. 2014, pp. 5-62. doi: $10.1017 / \mathrm{jfm} .2014 .505$

[39] Morgan, B., Duraisamy, K., Nguyen, N., Kawai, S., and Lele, S. K., "Flow Physics and RANS Modelling of Oblique Shock/Turbulent Boundary Layer Interaction,” Journal of Fluid Mechanics, Vol. 729, Aug. 2013, pp. 231-284. doi:10.1017/jfm.2013.301

[40] Martin, M. P., "Direct Numerical Simulation of Hypersonic Turbulent Boundary Layers. Part 1. Initialization and Comparison with Experiments," Journal of Fluid Mechanics, Vol. 570, Jan. 2007, pp. 347-364. doi:10.1017/S0022112006003107

[41] Pirozzoli, S., Bernardini, M., and Grasso, F., "Direct Numerical Simulation of Transonic Shock/Boundary Layer Interaction Under Conditions of Incipient Separation," Journal of Fluid Mechanics, Vol. 657, Aug. 2010, pp. 361-393. doi:10.1017/S0022112010001710

[42] Koo, H., Donde, P., and Raman, V., "A Quadrature-Based LES/ Transported Probability Density Function Approach for Modeling Supersonic Combustion," Proceedings of the Combustion Institute, Vol. 33, No. 2, 2011, pp. 2203-2210.

[43] Koo, H., Donde, P., and Raman, V., "LES-Based Eulerian PDF Approach for the Simulation of Scramjet Combustors," Proceedings of the Combustion Institute, Vol. 34, No. 2, 2013, pp. 2093-2100.

[44] Donde, P., Koo, H., and Raman, V., "A Multivariate Quadrature Based Moment Method for LES Based Modeling of Supersonic Combustion," Journal of Computational Physics, Vol. 231, No. 17, 2012, pp. 5805-5821. doi:10.1016/j.jcp.2012.04.031
[45] Fiévet, R., Voelkel, S., Koo, H., Raman, V., and Varghese, P. L., "Effect of Thermal Nonequilibrium on Ignition in Scramjet Combustors," Proceedings of the Combustion Institute, Vol. 36, No. 2, 2017, pp. 2901-2910.

[46] Koo, H., "Large-Eddy Simulations of Scramjet Engines," Ph.D. Thesis, Univ. of Texas at Austin, Austin, TX, 2011.

[47] Jiang, G.-S., and Shu, C.-W., "Efficient Implementation of Weighted ENO Schemes," Journal of Computational Physics, Vol. 126, No. 1, 1996, pp. 202-228. doi:10.1006/jeph.1996.0130

[48] Wagner, C., Huttl, T., and Sagaut, P., Large-Eddy Simulation for Acoustics, Cambridge Univ. Press, Cambridge, U.K., 2007, pp. 105-107.

[49] Clemens, N. T., and Narayanaswamy, V., "Low-Frequency Unsteadiness of Shock Wave/Turbulent Boundary Layer Interactions," Annual Review of Fluid Mechanics, Vol. 46, 2014, pp. 469-492. doi:10.1146/annurev-fluid-010313-141346

[50] Wu, M., and Martin, M. P., "Direct Numerical Simulation of Supersonic Turbulent Boundary Layer over a Compression Ramp," AIAA Journal, Vol. 45, No. 4, 2007, pp. 879-889. doi: 10.2514/1.27021

[51] Agostini, L., Larcheveque, L., and Dupont, P., "Mechanism of Shock Unsteadiness in Separated Shock/Boundary-Layer Interactions," Physics of Fluids, Vol. 27, No. 12, Dec. 2015, Paper 126103. doi: $10.1063 / 1.4937350$

J. Poggie Associate Editor 